\title{
Late - Onset Hypogonadism - New Point of View
}

\author{
Andrzej Gomuła* \\ The Andropause Institute, Warsaw, \\ Poland
}

\section{Introduction}

Long-term testosterone deficiency related to age may adversely affect health, anatomy and physiology of man. The implementation of testosterone boost therapy only at the time, when for many years, as the result of testosterone deficiency, irreversible anatomical changes have occurred, is clearly too late. Age-related progressive decrease in testosterone serum concentration levels causes anatomical and functional abnormalities. It is the cause of lipid disorders; it excarberates type-2 diabetes, it is also the common cause of cardiovascular diseases. It contributes to other health problems such as atherosclerosis, hypertension, osteoporosis and obesity and it manifests itself by decreased libido and potency. There is also a strong relationship between age-related decrease in testosterone and Parkinson's disease and Alzheimer's disease. Benign prostatic hyperplasia (BPH) and carcinoma of the prostate are closely associated with testosterone deficiency and comedo-carcinoma - the most malignant form of prostate cancer - is directly proportional to the decrease in serum testosterone. A good therapy for increasing testosterone serum levels can reverse the problems associated with aging such as type-2 diabetes, sexual dysfunction, osteoporosis, hyper-lipidemia and ischemic heart disease. It can even reverse symptoms of Parkinson's disease. Using synthetic testosterone is often recommended in the treatment of testosterone deficiency. Unfortunately, synthetic testosterone can cause side effects such as infertility and a long-term use of testosterone may also lead to irreversible testicular atrophy. Therefore, patients receiving long-term testosterone therapy are all dependent on adequate doses of synthetic testosterone until the end of their lives. Meanwhile, intramuscular administration of hCG to stimulate the endogenous testosterone synthesis, has been known since the 1950s. The induction of endogenous testosterone production by hCG has been effective in all age groups while being safe at the same time. In this paper, the author presents problems caused by testosterone deficiency and outlines the possibility of the treatment, which increases the induction of testosterone endosynthesis by hCG. It has not yet been determined how to diagnose testosterone deficiency. The age-related serum testosterone concentration reference range has not been established yet either. The paper presents the first attempt to establish international standards for testosterone serum concentration levels in different age groups.

\section{Late-onset hypogonadism}

Late-onset hypogonadism (LOH) is a testosterone deficiency syndrome resulting in the aging process. LOH leads to metabolic disorders and functional or anatomical abnormalities. It is

${ }^{*}$ Corresponding Author 
now beyond dispute that, as testosterone deficit increases with age, it adversely affects the function of multiple organ systems. Therefore the implementation of appropriate strategies to increase testosterone levels only when as a result of testosterone deficiency irreversible anatomical changes have occurred, is clearly too late, which operates to the detriment of men.

Recent studies suggest that there are large numbers of men in the community whose testosterone deficiency is neither being diagnosed nor treated (Trinick et al., 2011).

Testosterone exerts influence on multiple life processes such as blood cell production, bone formation, lipid metabolism, protein metabolism, carbohydrate metabolism, liver function, and spermiogenesis (Gooren, 2000). This can affect the structure and function of many organs in human body. They include skin, hair, muscle, brain and bones. It also has a significant effect on fertility and sexual behaviour. Testosterone plays a very important role in a man's life. At the mitochondrial level of each single cell it is the catalyst for protein synthesis. It has an effect on the brain's function, and thus determines the physical and sexual condition. It increases libido and improves sexual potency. It stimulates the immune system and affects multiple metabolic processes. It reduces body fat accumulation. It can affect muscle mass and strength. It increases bone mass and accelerates wound healing. Testosterone is responsible for our memory processes. Computer memory circuits are manufactured using metal arranged on silicon. In the process of human memory, proteins are involved. The testosterone that catalyzes the synthesis of various proteins is also a catalyst for storage protein synthesis. Protein synthesis becomes less efficient with age, proteins formed are unstable. Their decay means that an old man remembers what was years ago and cannot remember what just happened. In the literature, there have been isolated reports about the role of testosterone in the aetiology of Alzheimer's disease (Hogervorst, 2004).

Testosterone also aids in immune system protein synthesis, which protects us against infection. Hormone intake in pregnant women during the first trimester of pregnancy significantly affects hormone balance in young men. Although hormone therapy allows the woman to keep her pregnancy from failing, it is also essential for organogenesis, and in particular for the reproductive organs of male foetuses. According to the definition in Standards of Endocrinology, "Testosterone deficit disorder manifests itself in a decrease in libido and potency, in constant fatigue, in deterioration of mood and in sleep quality, in nervousness, in hot flushes, in low testosterone, and in elevated serum gonadotropin concentrations" (Zgliczyński \& Zgliczyński, 2002). However, according to the definition provided by ISSAM, the International Society of Andrology (ISA) and the European Association of Urology (EAU), it is "a clinical and biochemical syndrome associated with advancing age, characterized by typical symptoms and a deficiency in serum testosterone levels. It can cause a significant deterioration in over-all quality of life and adversely affect the function of many different organs and systems" (Nieschlag, et al., 2005). It is now beyond dispute that, as testosterone deficit increases with age, it adversely affects the function of multiple organ systems. Previously adopted minimum standard testosterone levels, at which there has been best evidence for treatment, are far too low. The implementation of the therapy to boost testosterone levels at the time when for many years, as a result of testosterone deficiency, irreversible anatomical changes have occurred, is clearly too late, which operates to the detriment of men (Gomuła \& Rabijewski, 2010). According to Tenover, if we consider the norm based on the level of total testosterone, in a group of 55-year-old men, $20 \%$ of them will be hypogonadal; and in the same group of men, when we adopt a standard based on the level of bio-available testosterone, hypogonadism will be diagnosed in half of them (Tenover, 1997). Cross-sectional studies have shown that, since 
this is associated with a simultaneous increase of SHBG levels, bioavailable testosterone may decline more significantly than apparent total testosterone (Snyder, 2001, Vermeulen, 2001). Only the testosterone circulating in the body which is not bound to SHBG is biologically active in the target organs. Therefore the measurement of bioavailable testosterone levels more accurately reflects a patient's clinical status than the measurement of total testosterone levels. The study of 810 men aged 24-90 years showed a strong correlation between age and a decrease in bioavailable testosterone level and in estradiol level $(r=-0.52)$. It was still strong when other variables were taken into account such as alcohol intake, BMI, smoking, caffeine intake, and diabetes (Ferrini \& Barrett-Connor, 1998). Also significant but weaker $(r=-0.13)$ correlations were found between age and total testosterone and estradiol levels. Both the decreased testosterone production and its increased conversion can explain a reduction in total testosterone levels, while age-related increase in sex hormone binding capacity can cause reduced biologically available testosterone production. The correlation between age and the level of bioavailable and of total estradiol in men has not been studied previously. Bioavailable estradiol levels might decrease due to a decline in levels of testosterone, which is the main substrate for male estradiol production. It also contributes to an increase of SHBG with age. In one of the largest studies, which involved 2,623 men aged 65 and over, enjoying good health, levels of free and bioavailable testosterone and estradiol levels correlated with each other (Orwoll, et al. 2006). Higher age, higher BMI, and worse health status were associated with slightly lower total testosterone levels. The concentration of SHBG rose with age. The decrease in testosterone levels fell approximately $10 \%$ for 10 years. Many old men still had testosterone levels established for young men. However, it is unknown which level of testosterone is sufficient for an elderly person and for that person's specific tissues (i.e. muscles, bones, and nervous system). The number of androgen binding sites in the hippocampus and the number of the layers of the skin tissues that cover the penis increase with age (Hijazi \& Cunningham, 2005). Testosterone production peaks at age 20 and then begins a gradual decline, usually giving rise to the first symptoms after the age of 50. In a group of men aged 80, the level of free testosterone is half of what they had when they were younger (Vermeulen, et al., 1996).

\subsection{Hypogonadism - Primary, secondary, LOH}

Classic categorization of hypogonadism differentiates its primary and secondary types. In the case of primary hypogonadism, testicles are unable to synthesize estosterone, while in case of secondary hypogonadism low testosterone is caused by pituitary insufficiency. Until present from the pathophysiology point of view, LOH has been regarded as a mixed type of hypogonadism conditioned by the changes undergoing both in gonads (component of primary hypogonadism) and on the level of central controllers of testicular functions; hypothalamus and pituitary (components of secondary hypogonadism). Hypothalamus and pituitary regulate, on the basis of feedback, functions of Leydig cells in testicles. Gonadotrophin-releasing hormone is a trophin hypothalamus hormone relative to the Leydig cells. Its effect on pituitary results in the synthesis of luteinizing hormone (LH) and folicule stimulating hormone (FSH) in gonadotrophin cells. Gonadotrophin LH stimulates the synthesis of testosterone through the Leydig cells in testicle. With age, pulsating secretion of LH becomes disturbed. Pulses become more scarce (decreased frequency), of lower amplitude and their duration extends. The result is an increase of LH concentration with age. If LH concentration increases with age, thus pituitary insufficiency, i.e. secondary hypogonadism, does not exist and testicles retain their capability of high endosynthesis of testosterone, hence in case of LOH features of primary or of secondary hypogonadism are 
non-existent. It has been acknowledged that testicle stimulation with hCG does not cause any substantial, sufficient increase of endosynthesis of testosterone. And here lies the fundamental error - the statement that hCG does not result in any substantial increase of testosterone endosynthesis is untrue!!! Therapy based on hCG allows doubling the concentration of testosterone, which in over $90 \%$ of those treated enables restoring a normal hormone condition. I base my statement on over a 10-year long observation of 1200 patients I treated with hCG to induce endosynthesis of testosterone.

Secretion of GnRH is distorted as well. Hypothalamus response to change of LH pulse nature and to lowered secretion of androgens is incommensurate. The phenomenon is referred to as dysregulation of hypothalamus pulse generator. The reasons for alternations of pulsating secretion of $\mathrm{GnRH}$ and $\mathrm{LH}$ are unclear, however of importance are genetic factors and backward changes in gonadotrophin cells and in reflective microcirculation on hypothalamus and pituitary axis (Snyder, et al., 2000). Ferrini, Wang, Hakim et al. have established that changes in functioning of hypothalamus and pituitary may not be the major cause of gonads dysfunctions in men (Ferrini, et al., 2001). They have found evidence for a substantial increase of hypothalamus and pituitary apoptosis, two changes clarifying development of hypogonadism at old age. Until now it was believed that together with hypothalamus and pituitary dysfunction gonadal backward changes played an important role in etiopathogenesis of gonads. The process of decreasing testosterone synthesis starts approximately at the age of 35-40 years and is of extreme individual variability. Changes within testicles are noted for progressive decrease of the number of Leydig cells and deterioration of Sertoli cells functions and as a result decrease of secretion of inhibin, impaired micro-circulation and blood-supply of testicle cells and backward changes within perivascular parenchyma (Deslypere \& Vermulen, 1984, Snyder, 2001). From the analysis of own material it is considered that to-date views on TDS aetiology are unfounded. As it has been demonstrated in the material consisting of 908 men treated with induction of endosynthesis, it is possible with every age group, but it decreas in terms of physiology with age. Nevertheless, the capability of efficient endosynthesis is retained until advanced years, which contradicts views of atrophy of Leydig cells functions. From a clinical point of view, LOH resembles pathology of incorrect LH bioactivity described in men of 47XY karyotype who after adolescence undergo hypogonadism with decrease of testosterone and increase of LH concentration. These patients demonstrate correct gonads response to LH stimulation. It has been acknowledged that in these cases LH bioactivity is decreased, while immunoreactivity is normal. It results from glutamine changing into arginine in position 54 of beta chains.

Currently, it is not possible to define explicitly the aetiologic factor of $\mathrm{LOH}$ leading to testosterone shortfall. Testosterone shortfall results in metabolic syndrome causing disorders of many bodily functions such as memory disorders, difficulties with falling asleep, excessive nervousness, deterioration of ability to associate and concentrate, heat waves, depression tendencies, vertigo and headache, fall of strength and muscle bulk, as well as numerous cardiovascular system complaints. Testosterone concentration drop leads to lipid disorders and diabetes type 2 .

\subsection{Andropause, ADAM, PADAM, LOH, TDS}

The concept of "male menopause" has been known since 1960s (Wang, et al., 1996) yet until today it has not been unambiguously defined. The names of the clinical state caused by testosterone shortfall have changed many times over decades which explicitly proves lack of firm facts based on indisputable ground. Names of andropause, sarcopenia, viropause were 
followed by andropause, (Morley \& Perry, 2003) which stayed in medicine for good. Many learned societies with the term of andropause in the centre were established around the world. However the term of andropause is erroneous by definition. Such a phenomenon as pause, break in production of testosterone with men does not exist. Since a pause is a stage of an activity as discontinuation and a pause (a break) is followed by return to the activity from before the pause. At the end of previous century notions of Androgen Deficiency in Aging Male or Androgen Deficiency in Adult Male /ADAM/ (Carruthers, 2004) came to being. It must be emphasized that an adult man is aged between 20-25 years, while an aging man according to International Society for the Study of the Aging Male (ISSAM) is a gentleman above 60-65 (Carruthers, 2004). This is where the dilemma arises, does the testosterone shortfall issue concern exclusively elder men or younger, adult men? Testosterone shortfall has earned yet another name- PADAM (partial androgen deficiency in aging male), which defines the testosterone shortfall in a not sufficiently explicit manner. The concept lacks specification of boarder testosterone concentration and thus the value according to which a shortfall can be recognized. Therefore the term PADAM should not be used at all. What does partial shortfall mean? No accounting system recognizes partial deficit, it either exists or does not. Can a woman be partially pregnant? Another term was established; secondary or late onset hypogonadism but it still did not represent the core of the problem (Morales, et al., 2006). As clinical symptoms of androgen shortfall manifest themselves already at the break of forth and fifth decade (Mosby, 1998) (35-45 years), i.e. in the first half of life, can it be said that hypogonadism appears late? The latest name of the disease, which so far has not been explicitly defined, is testosterone deficiency syndrome /TDS / (Morales, et al., 2006) currently a fashionable term. Yet science and fashion follow totally different criteria. What counts in fashion is a one-season success, while in science exact, indisputable facts forming a solid basis for many years should be essecial. In the meantime, testosterone shortfall, an unfavourable phenomenon leading to various metabolic disorders or issue pathologies, has not been named a disease until now.

\subsection{Late - onset hypogonadism and age related testosterone levels}

Many studies have now demonstrated that as men age, their serum testosterone concentrations fall at an average rate of $0.8 \%-1 \%$ per year (Feldman, et al., 2002, Kaufmann \& Vermeulen, 2005). Concurrently, free and bioavailable testosterone levels fall by $2 \%$ per year (Kaufmann \& Vermeulen, 2005). Obesity, alcohol abuse, diabetes, hypertension, heart disease, cancer and ulcers have intensified the negative impact (Harman, et al., 2001).

Therefore, and also due to an increase in the concentrations of carrier proteins, significantly decreased testosterone levels characterise approximately $8 \%$ of men of $40-60$ and $20 \%$ men of 60-80 (Kaufmann \& Vermeulen, 2005). The question arises: how to measure the decrease in testosterone levels during the life of a man? By the hormonal status of young people today? The hormonal status of their fathers? What was the hormonal status of their grandparents in their youth, since testosterone levels in men over age 70 often reach the upper range of the standard? And another question: what was the testosterone level of a 30year-old man, whose level now, at 70, is more than $35 \mathrm{nmol} / \mathrm{L}$ ?

Standard testosterone levels vary in different analytical laboratories. In American research laboratories alone, the lower limit of normal testosterone level ranges from 4.5 to 15.6 $\mathrm{nmol} / \mathrm{L}-350 \%$ difference (Lazarou, et al., 2006)

The effects of an objectively measurable drop in testosterone levels may also vary. Researchers wonder if it would be most appropriate to establish separate ranges of normal testosterone 
levels for the young and for the old (Lunenfeld \& Gooren, 2002). It is also unknown whether the plasma testosterone threshold values vary with age. There is preliminary evidence that the threshold value necessary for the proper functioning and for the proper effects of testosterone also increases with age (Schiavi, et al., 1993). Bancroft hypothesizes that the threshold required for the behavioral effects of testosterone increases with age (Bancroft, 1989). Although many older men have testosterone levels within the normal range, their levels may not be sufficient for normal sexual functioning. Probably organ sensitivity to androgens is not the same for the young and for the old. Schiavi reported that nocturnal erections are androgen-dependent and are disrupted in healthy elderly men who do not meet the criteria for hypogonadism, which seems to confirm Bancroft's hypothesis (Bancroft, 1989, Schiavi, et al. (1993). Based on the analysis of the material provided by 1267 men of 20-89, together with the material provided by 908 men under treatment, standard levels of testosterone have been age-determined (Gomuła \& Rabijewski, 2010). Among the respondents between 20-29, the average level of testosterone was $21.99 \mathrm{nmol} / \mathrm{L}$; among the respondents between 30-39 - $21.91 \mathrm{nmol} / \mathrm{L}$ among the respondents between 40-49 - $19.18 \mathrm{nmol} / \mathrm{L}$; among the respondents between 40-49 - 19.18 $\mathrm{nmol} / \mathrm{L}$; among the respondents between 50-59 - $17.74 \mathrm{nmol} / \mathrm{L}$; among the respondents between 60-69 - $15.94 \mathrm{nmol} / \mathrm{L}$ among the respondents between 70-79 - $17.66 \mathrm{nmol} / \mathrm{L}$ and among the respondents between 80-89 - $16.65 \mathrm{nmol} / \mathrm{l}$. The standard testosterone levels in individual age groups (minimum and maximum, average level, standard deviations and medians) are shown in Table 1.

\begin{tabular}{|c|c|c|c|}
\hline $\begin{array}{l}\text { Age/min-max, } \\
\text { median,average,SD }\end{array}$ & $\begin{array}{l}\text { n, Testosterone } \\
\text { in nmol/L }\end{array}$ & $\begin{array}{l}\text { Age/min-max, } \\
\text { median,average,SD }\end{array}$ & $\begin{array}{l}\text { n, Testosterone } \\
\text { in } \mathrm{nmol} / \mathrm{L}\end{array}$ \\
\hline 20-29 years & $\mathrm{n}=78$ & $60-69$ years & $\mathrm{n}=281$ \\
\hline Min., max. & $4.64 ; 49.10$ & Min., max. & $2 ; 40.40$ \\
\hline Average (SD) & $21.99(9.33)$ & Average (SD) & $15.94(6.68)$ \\
\hline Median $(25 \%, 75 \%)$ & $19.75(15.40 ; 26.20)$ & Median $(25 \%, 75 \%)$ & $15(11.30 ; 19.70)$ \\
\hline $30-39$ years & $\mathrm{n}=102$ & $70-79$ years & $\mathrm{n}=213$ \\
\hline Min., max. & $8.90 ; 46.80$ & Min., max. & $2.71 ; 45.60$ \\
\hline Average (SD) & $21.91(8.18)$ & Average (SD) & $17.66(7.87)$ \\
\hline Median $(25 \%, 75 \%)$ & $21.70(15.80 ; 25.20)$ & Median $(25 \%, 75 \%)$ & $16.10(12.70 ; 21.30)$ \\
\hline $40-49$ years & $\mathrm{n}=176$ & $80-89$ years & $\mathrm{n}=50$ \\
\hline Min., max. & $3.40 ; 47.20$ & Min., max. & $3.70 ; 38.80$ \\
\hline Average (SD) & 19.18 (8.92) & Average (SD) & 16.65 (7.55) \\
\hline Median $(25 \%, 75 \%)$ & $17(12.6 ; 24.30)$ & Median $(25 \%, 75 \%)$ & $15.75(12 ; 20.60)$ \\
\hline $50-59$ years & $\mathrm{n}=367$ & & \\
\hline Min., max. & $3.10 ; 53.50$ & & \\
\hline Average (SD) & $17.74(8.01)$ & & \\
\hline Median $(25 \%, 75 \%)$ & $16.20(12.10 ; 21.70)$ & & \\
\hline
\end{tabular}

$\mathrm{n}$ - number of patients in the age group, SD - standard deviation

Table 1. Testosterone levels of 1267 men in different age groups 
The evaluation of 1267 men aged 20-89 years has found that the average testosterone level in all age groups is higher than $12 \mathrm{nmol} / \mathrm{L}-$ what is shown in Fig. 1.

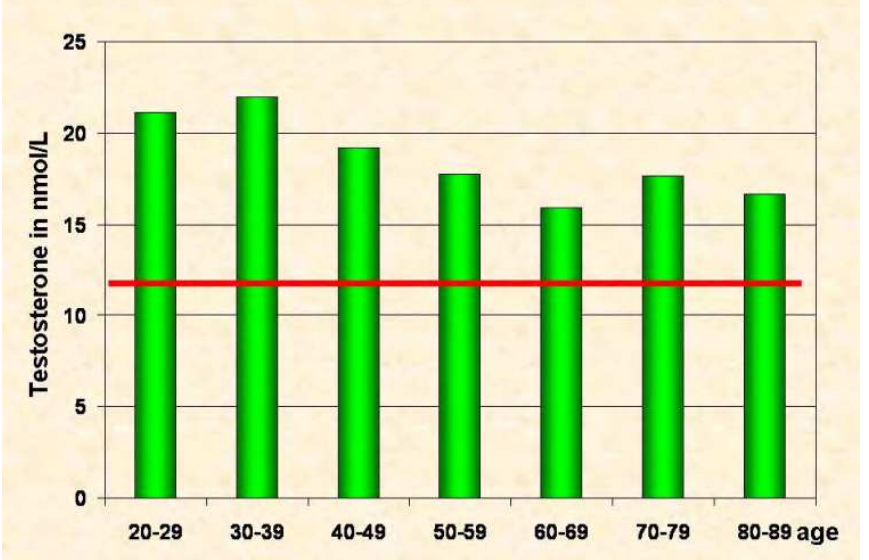

Fig. 1. The average testosterone levels of 1267 men in different age groups. The horizontal line on the graph represents an accepted lower limit of normal testosterone level $12 \mathrm{nmol} / \mathrm{L}$ / according to the International Society for the Study of the Aging Male/ (Lunenfeld, B. Et al., 2005, Nieschlag, et al., 2005). If we assume that the standard testosterone level is higher than $12 \mathrm{nmol} / \mathrm{L}$, in the age group 20-29 years testosterone deficit has been found in $11.5 \%$ of those examined. In the age group 30-39 years the deficit has been found in $5.9 \%$ of those examined. In the age group $40-49$ years - in $21 \%$ of those examined; in the age group $50-59$ years - in $24 \%$ of those examined; in the age group $60-69$ years - in $27.8 \%$; in the age group $70-79$ years - in $20.6 \%$; and in the age group $80-89$ years - in $24 \%$ of those examined.

Testosterone deficiency (serum testosterone concentrations below $12 \mathrm{nmol} / \mathrm{L}$ ) in different age groups is shown in Table 2.

\begin{tabular}{|c|c|c|}
\hline Age & N pts & \% \\
\hline $20-29$ & $9 / 78$ & $11.5 \%$ \\
\hline $30-39$ & $6 / 102$ & $5.9 \%$ \\
\hline $40-49$ & $37 / 176$ & $21 \%$ \\
\hline $50-59$ & $88 / 367$ & $24 \%$ \\
\hline $60-69$ & $78 / 281$ & $27.8 \%$ \\
\hline $70-79$ & $44 / 213$ & $20.6 \%$ \\
\hline $80-89$ & $12 / 50$ & $24 \%$ \\
\hline
\end{tabular}

Table 2. Testosterone deficiency in different age groups.

On the basis of the TDI ratio, testosterone deficiency has been found in $49.55 \%$ of those in the study group. The smaller number of patients who had TDI determined is due to the absence of the ratio of LH for 48 out of 1267 patients. Summary of parameters to provide a basic index of the Andropause status for 1219 patients is shown in Table 3. 


\begin{tabular}{|l|}
\hline \multicolumn{1}{|c|}{ Parameter $\mathbf{n}=\mathbf{1 2 1 9}$ pts } \\
\hline \multicolumn{1}{|c|}{ T (ng/mL) } \\
\hline Min., max. $=0.58 ; 15.41$ \\
Average (SD) $=5.22(2.33)$ \\
Median $(25 \%, 75 \%)=4.78(3.59 ; 6.48)$ \\
\hline \multicolumn{1}{|c|}{ LH (IU/L) } \\
\hline Min., max. = 0.2; 47 \\
Average (SD) = 5.8 (4.6) \\
Median $(25 \%, 75 \%)=4.8(3.3 ; 6.7)$ \\
\hline \multicolumn{1}{|c|}{ T/LH } \\
\hline Min., $\operatorname{max.}=0.02 ; 41.76$ \\
Average (SD) $=1.36(1.67)$ \\
Median $(25 \%, 75 \%)=1(0.63 ; 1.61)$ \\
\hline TDI < 1 = 604/1219 (49.55\%) \\
\hline
\end{tabular}

$\mathrm{T}$ - testosterone; $\mathrm{LH}$ - luteinizing hormone; $\mathrm{SD}$ - standard deviation; TDI - testosterone deficit index.

Table 3. Average serum testosterone concentrations , LH and TDI

The findings in Table 4 show, on the basis of the TDI ratio, an increased incidence of testosterone deficiency with age.

\begin{tabular}{|cc|}
\hline Age & TDI < 1 \\
\hline $20-29$ & $21 / 78(28 \%)$ \\
$30-39$ & $29 / 101(28.7 \%)$ \\
$40-49$ & $56 / 165(33.9 \%)$ \\
$50-59$ & $160 / 355(45.1 \%)$ \\
$60-69$ & $174 / 271(64.2 \%)$ \\
$70-79$ & $124 / 197(62.9 \%)$ \\
$80-89$ & $38 / 47(80.85 \%)$ \\
\hline
\end{tabular}

Table 4. Testosterone deficiency in different age groups for 1219 male respondents, calculated on the basis of TDI.

Two methods of assessing testosterone deficiency have been compared, based on global standards and on TDI. Figures 2 and 3 show the material discussed above under analysis. In the group of 1267 patients, there were only 274 males with serum testosterone concentrations levels below $12 \mathrm{nmol} / \mathrm{L}$, which amounts to $21.62 \%$ of those examined, whereas, in accordance with TDI standards, 604 out of 1219 men had testosterone deficiency, which amounts to $49.55 \%$ of those examined. The observed difference between the groups is statistically significant.

If we put on the two graphs (Figure 2 and Figure 3) the lines connecting testosterone level in young men when they have the highest testosterone level (i.e., 30-39 year olds), it turns out that the testosterone deficit estimation results in accordance with the standardised testosterone-below-12-nmol/1 approach are different from those in accordance with TDI standards. As shown in Figure 2, in accordance with the most rigorous standards of serum testosterone concentration, men aged 60-70 years have been diagnosed with testosterone 


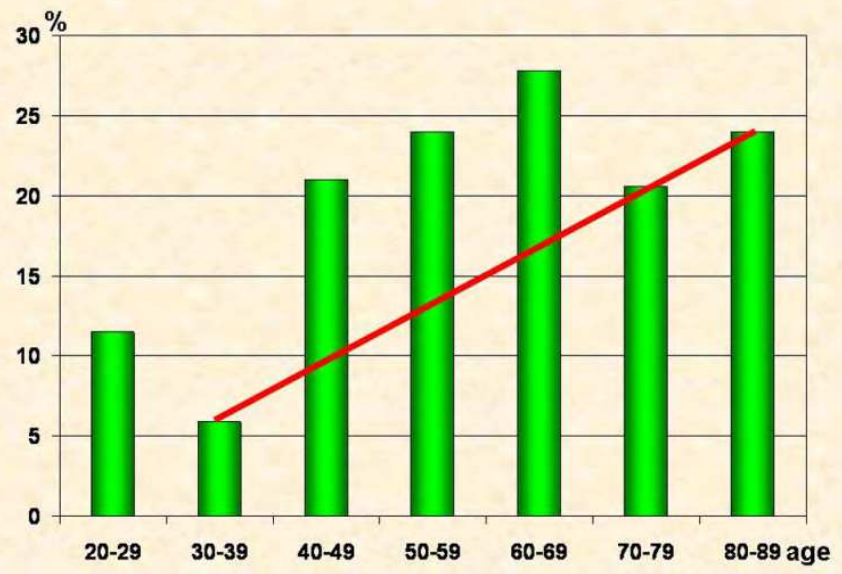

Fig. 2. Testosterone levels below $12 \mathrm{nmol} / \mathrm{L}$ in relation to age.

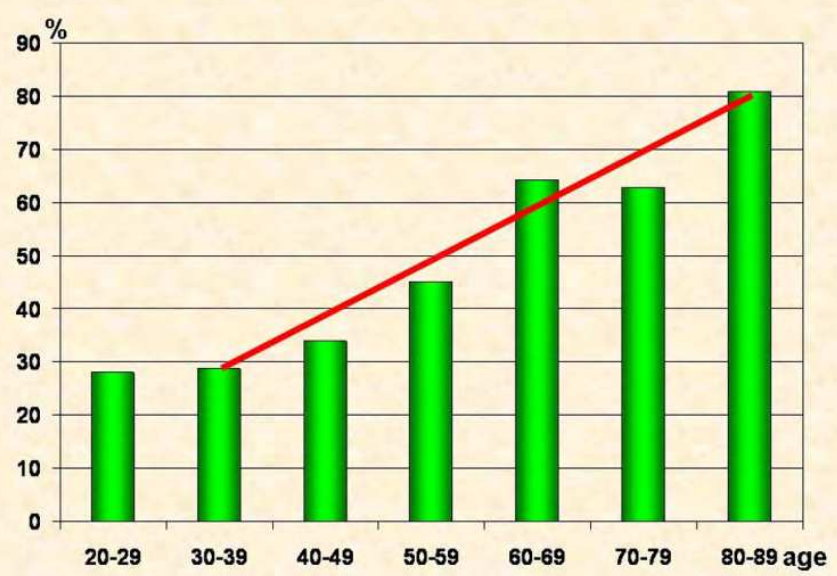

Fig. 3. TDI (Testosterone Deficit Index) in relation to age.

deficiency, while testosterone deficiency levels have clearly been lower in the age group 7079 years and in the age group 80-89 years. An assessment in accordance with the testosterone-concentrations-below-12-nmol/L parameter exhibits significant deviations. It shows that, as men age, testosterone deficiency does not increase in a linear fashion. However, Figure 3 shows that the analysis of the same material on the basis of the testosterone deficiency index (TDI) demonstrates that, as men age, testosterone deficiency increases in a linear fashion. The study proves the effectiveness of the testosterone deficiency index (TDI) in assessing the degree of testosterone deficiency. On the basis of the TDI results and/or on the basis of a patient's clinical status, 903 of those examined were involved in testosterone endosynthesis induction treatment with human chorionic gonadotropin /hCG/ (Gould, 1951, Gomula, 2001, Gomula, 2002). Testosterone levels in response to hCG treatment are shown in Table 5, and graphically in Figure 4. 


\begin{tabular}{|c|c|c|c|c|}
\hline Age & T-0 nmol/L & T-1 nmol/L & Change & $\mathrm{p}$ \\
\hline $20-29$ & $\mathrm{n}=44$ & $\mathrm{n}=44$ & $\mathrm{n}=44$ & \\
\hline Min., max. & $6.2,49.1$ & $20.30,89.10$ & $1.5,42.2$ & \\
\hline Average (SD) & $21.13(9.7)$ & $44.03(13.45)$ & $22.9(10.9)$ & \\
\hline $\begin{array}{l}\text { Median }(25 \%, \\
75 \%)\end{array}$ & $\begin{array}{l}19.7(14.05 \\
24.55)\end{array}$ & $\begin{array}{l}44.05(34.70 \\
51.15)\end{array}$ & $\begin{array}{l}23.55(14.35 \\
30.9)\end{array}$ & $<0.001$ \\
\hline $30-39$ & $n=63$ & $\mathrm{n}=63$ & $\mathrm{n}=63$ & \\
\hline $\begin{array}{l}\text { Min., max. } \\
\text { Average (SD) } \\
\text { Median (25\%, } \\
75 \%)\end{array}$ & $\begin{array}{l}8.9 ; 46,8 \\
21.78(8.31) \\
21.7(15.9 ; 24)\end{array}$ & $\begin{array}{l}20.50 ; 96.20 \\
49.62(16.12) \\
46.80(37.60 ; \\
60.40)\end{array}$ & $\begin{array}{l}3.9 ; 63.6 \\
27.8(13.5) \\
28.9(16.6 ; 35.7)\end{array}$ & \\
\hline $40-49$ & $n=131$ & $\mathrm{n}=131$ & $\mathrm{n}=131$ & \\
\hline $\begin{array}{l}\text { Min., max. } \\
\text { Average (SD) } \\
\text { Median (25\%, } \\
75 \%)\end{array}$ & $\begin{array}{l}6.3 ; 46.6 \\
18.5(8.47) \\
16.5(12.3 ; 23.6)\end{array}$ & $\begin{array}{l}11.40 ; 78.20 \\
41.72(14.36) \\
39.60(31.60 ; \\
50.50)\end{array}$ & $\begin{array}{l}2.1 ; 58.5 \\
23.2(12.09) \\
23.13(13.8 ; 32.6)\end{array}$ & $<0.001$ \\
\hline $50-59$ & $\mathrm{n}=277$ & $\mathrm{n}=277$ & $\mathrm{n}=277$ & \\
\hline $\begin{array}{l}\text { Min., max. } \\
\text { Average (SD) } \\
\text { Median (25\%, } \\
75 \%)\end{array}$ & $\begin{array}{l}3.1 ; 46.4 \\
17.14(7.72) \\
15.7(11.9 ; 20.5)\end{array}$ & $\begin{array}{l}10.30 ; 78.30 \\
39.23(12.63) \\
38.50(29.80 ; \\
46.20)\end{array}$ & $\begin{array}{l}4.9 ; 62.6 \\
22.08(11.7) \\
20.1(13.7 ; 29.6)\end{array}$ & $<0.001$ \\
\hline $60-69$ & $\mathrm{n}=205$ & $\mathrm{n}=205$ & $n=205$ & \\
\hline $\begin{array}{l}\text { Min., max. } \\
\text { Average (SD) } \\
\text { Median (25\%, } \\
75 \%)\end{array}$ & $\begin{array}{l}2.71 ; 40.4 \\
15.59(6.45) \\
14.4(10.8 ; 19.1)\end{array}$ & $\begin{array}{l}7.50 ; 82.30 \\
34.48(13.32) \\
32.30(24.70 ; \\
41.90)\end{array}$ & $\begin{array}{l}4.3,69.2 \\
18.9(11, .9) \\
16.8(10.6 ; 25)\end{array}$ & $<0.001$ \\
\hline $70-79$ & $\mathrm{n}=154$ & $\mathrm{n}=154$ & $\mathrm{n}=154$ & \\
\hline $\begin{array}{l}\text { Min., max. } \\
\text { Average (SD) } \\
\text { Median (25\%, } \\
75 \%)\end{array}$ & $\begin{array}{l}2.71 ; 45.6 \\
17.09(7.5) \\
15.15(12.5 ; 19.9)\end{array}$ & $\begin{array}{l}6.80 ; 89 \\
34.08(14.83) \\
32(23.50 ; 42.70)\end{array}$ & $\begin{array}{l}7.2 ; 59 \\
16.99(13.32) \\
15.6(6.3 ; 24.2)\end{array}$ & $<0.001$ \\
\hline $80-89$ & $\mathrm{n}=29$ & $\mathrm{n}=29$ & $\mathrm{n}=29$ & \\
\hline $\begin{array}{l}\text { Min., max. } \\
\text { Average (SD) } \\
\text { Median (25\%, } \\
75 \%)\end{array}$ & $\begin{array}{l}3.7 ; 27.8 \\
14.39(5.43) \\
14.8(12 ; 17.6)\end{array}$ & $\begin{array}{l}11,50 ; 48,50 \\
27.10(10.36) \\
24.50(18.80 ; \\
35.40)\end{array}$ & $\begin{array}{l}4.9 ; 29.6 \\
12.72(9.86) \\
12.4(5.4 ; 19)\end{array}$ & $<0.001$ \\
\hline
\end{tabular}

Table 5. Testosterone concentration before (T-0) and during hCG treatment (T-1) in different age groups.

Using Student's t-test or the Wilcoxon rank-sum test, it has been examined whether the testosterone concentration change is significantly different from 0 . Statistically significant results have been achieved both for the whole group and when broken down by age group. 


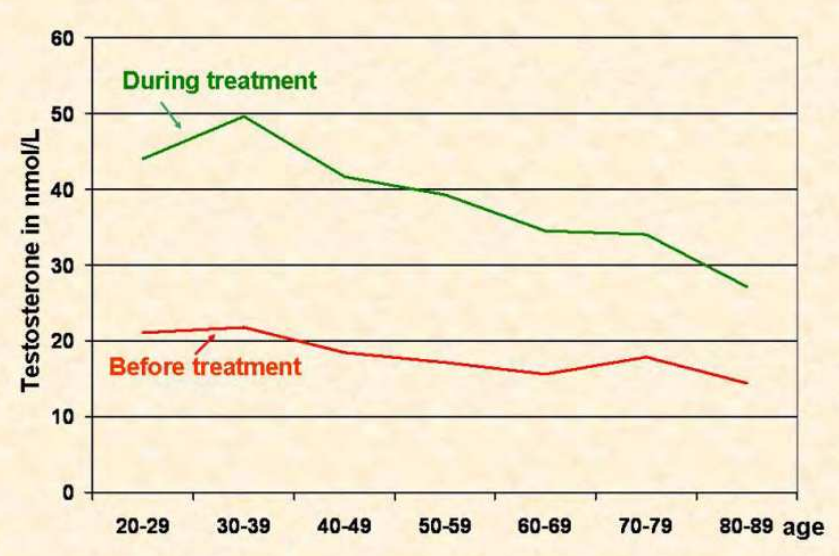

Fig. 4. Testosterone levels in different age groups before (the lower, red line) and during hCG treatment (the upper, green line).

Testosterone concentrations in different age groups have risen for 908 patients under hormone therapy (testosterone endosynthesis induction treatment through the administration of hCG) by an average of between 88 percent and 128 percent. At the same time, it has been found that under hCG stimulation the prospects of endosynthesis decrease linearly with age in the age group 30-89 years. This is consistent with physiology (Figure 5). The prospects of testosterone endosynthesis decrease with age. The age group $30-39$ years had an average $128 \%$ increase in testosterone levels; the age group 40-49 years - an average $126 \%$ increase in testosterone levels; the age group $50-59$ years - an average $129 \%$ increase in testosterone levels; the age group 6069 years - an average $121 \%$ increase in testosterone levels; the age group 70-79 years - an average $99 \%$ increase in testosterone levels; the age group $80-99$ years - an average $88 \%$ increase in testosterone levels. The full range of values of parameters determining the prospects of testosterone endosynthesis in different age groups has been shown in Table 5.

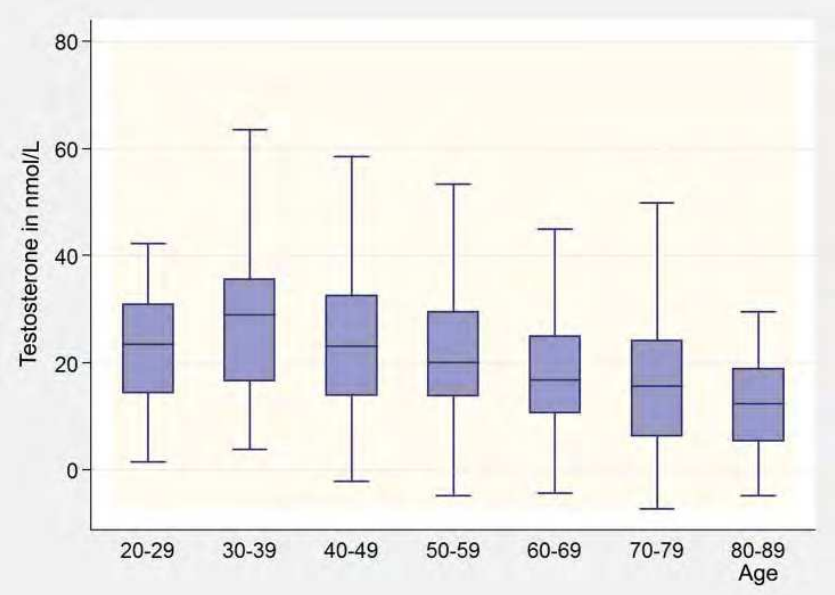

Fig. 5. The prospects of testosterone endosynthesis in different age groups. 
On the basis of the findings of the analysis of testosterone concentrations that occur physiologically, without treatment, during testosterone endosynthesis stimulation, it was decided to measure standard physiological testosterone levels correlated with age. During testosterone endosynthesis stimulation, testosterone concentrations obtained ade it possible for the patients to exhibit significantly better mood ratings, to develop improved physical fitness, and to increase libido and potency. The testosterone concentrations obtained also had the following positive impacts on blood pressure and biochemical parameters: normalization of the lipid profile and the reduction in HbA1clevels. Therefore, the testosterone concentrations obtained were adopted as normative in different age groups. Standard deviations of testosterone level increase while inducing testosterone endosynthesis by administering hCG varies by age group. This situation has been taken into account in measuring standard testosterone levels in different age groups. It has been assumed that normal testosterone levels in a particular age group is a number between the highest average value of testosterone while inducing testosterone endosynthesis by administering hCG and one standard deviation in the age group. These standards are illustrated graphically in Figure 6, and entered in Table 6.

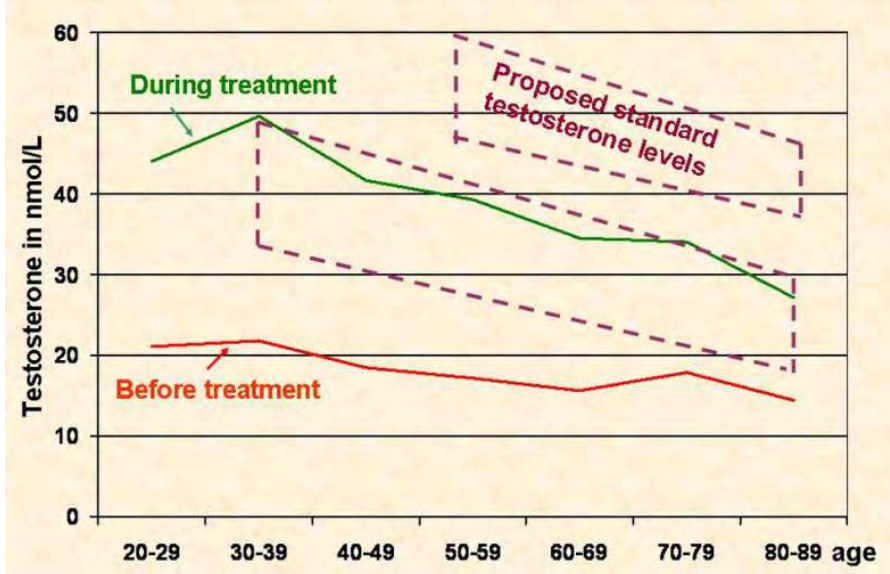

Fig. 6. The proposed standard testosterone levels for men of various age groups, based on the physiological capabilities caused by testosterone endosynthesis. The bottom line - the serum testosterone concentration in different age groups, prior to treatment; top line testosterone concentrations in each age group during hCG treatment; box - proposed agerelated standard testosterone levels.

\begin{tabular}{|c|c|c|c|c|c|c|}
\hline Age & $30-39 y$ & $40-49 y$ & $50-59 y$ & $60-69 y$ & $70-79 y$ & $80-89 y$ \\
\hline $\mathrm{T}(\mathrm{nmol} / \mathrm{lL})$ & $33.5 \div 49.6$ & $27.3 \div 41.7$ & $26.6 \div 39.2$ & $21 \div 34.4$ & $19.2 \div 34.0$ & $16.7 \div 27.1$ \\
\hline
\end{tabular}

Table 6. Standard testosterone levels for men of various age groups, based on the analysis of potential testosterone endosynthesis capabilities among 908 patients treated by inducing testosterone endosynthesis by administration of hCG. 
A very important question arises: if testosterone levels are so high, as the authors propose, are they not harmful? However, since these concentrations have been obtained by endosynthesis, it is obvious that they are in accordance with the body's physiological ability to synthesize testosterone. In addition, it has been known for more than 20 years that longterm high-dose testosterone therapy does not give significant metabolic side effects (Matsumoto, 1990). The correct threshold for proper testosterone functioning and for proper testosterone effects also increases with age (Schiavi, et al., 1993). Therefore the proposed age-related standards for testosterone must be much higher than those observed previously. A retrospective analysis of material possessed has suggested that the hormonal balance in today's young men is significantly worse than the hormonal balance for their fathers when they were in their youth. It has been shown in fact that the total testosterone level in today's young men is roughly two-thirds of their fathers' testosterone level. If we draw a line in parallel with the decline in serum testosterone levels with age for the age group of 35 to 70 years, and then draw it backwards, from the age of 90 to the earlier years, it will appear that the fathers, aged 30-35, exhibited significantly higher testosterone levels than their descendants at comparable ages (Fig. 7).

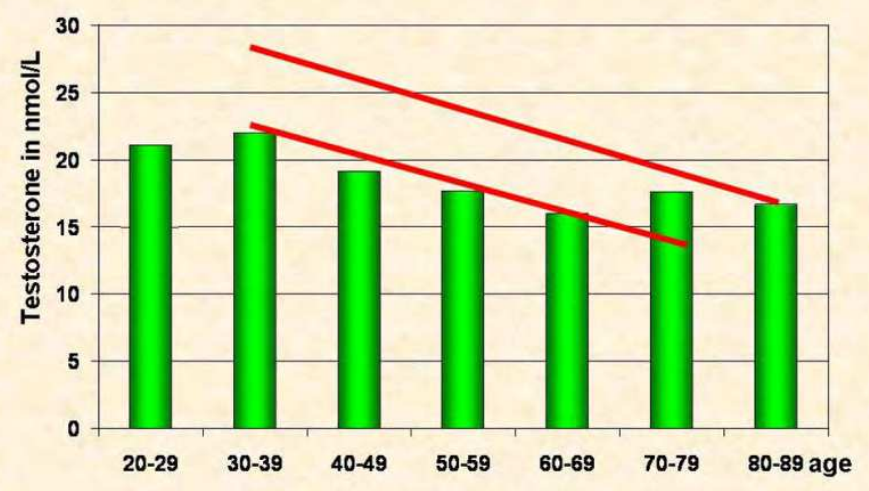

Fig. 7. Concentrations of testosterone in men aged 20-90 years and the line aptly showing their fathers' testosterone levels in the past.

Figure 7 shows that the concentration of testosterone in today's young men aged 30-35 is, on average, $23 \mathrm{nmol} / \mathrm{L}$ Concentrations of testosterone of their ancestors aged 85-89 averaged $17.2 \mathrm{nmol} / \mathrm{L}$ Therefore, standard values in the testosterone concentration range - the dispersion of values previously ranging to $31 \mathrm{nmol} / \mathrm{L}-$ have now narrowed to such an extent that the difference in testosterone levels between younger and older men is only 5 nmol/L i.e. $16 \%$ of normal range $10-42 \mathrm{nmol} / \mathrm{L}$ It practically means that young men now have testosterone levels about 35\% lower than those of their ancestors. Therefore, it can be concluded that, on the one hand, the whole world population is aging. On the other hand, the biological condition of today's youth suggests that in such a metabolic-hormonal state the youth of today will not increase human life expectancy. It is highly likely that they will die at a much younger age than their ancestors did in the past. The reasons for these changes are more complex. Genetic factors, lifestyle habits, medications, toxins, free radicals, body weight, psychological and social aspects, chronic stress, mental health, and social position play a significant role. 


\section{Late - onset hypogonadism and other diseases}

Testosterone levels in maturing man peak around age of 30 and then start to decline slowly. This decline in serum testosterone of aging men can lead to the development of many diseases (e.g. hypertension, ischemic heart disease etc.). The metabolic syndrome (defined as a combination of lipid abnormalities and cardiovascular risk factors), abdominal obesity, insulin resistance, arterial hypertension or raised blood pressure, are also associated with testosterone deficiency (Kalyani \& Dobs, 2007). All men are at risk for erectile dysfunction and prostate diseases including Benign Prostatic Hyperplasia (BPH) and Prostate Cancer (PC), which occur in later years of life of men. Additionally, Alzheimer's disease and Parkinson's disease are both common in the elderly men, especially in those over 85 . Low testosterone levels - a risk factor for development of visceral obesity - are associated with an acute decrease in circulating HDL cholesterol and increase of triglycerides. There may be a link between low testosterone levels in males and type 2 diabetes with elevated insulin levels. Some of these problems are due to the concentrations of total and free testosterone but there are also those that are associated with the derivatives of persistent testosterone deficiency. Late onset hypogonadism may result in the metabolic syndrome frequently leading to diabetes and/or to accelerated heart disease. When testosterone hormones in our body are balanced, the symptoms disappear. An increase in serum testosterone, results in normalization of lipid profile, improves glucose tolerance, decreases $\mathrm{HbA1c}$, and produces the increase in bone mass observed in densitometry. Pharmacological correction of hormones results in marked improvements in the sexual health (increasing both libido and potency), removes depression symptoms, and reduces or completely removes all symptoms of BPH and of Parkinson's disease.

\subsection{Late - onset hypogonadism is not just testosterone deficiency}

So far the aging process and clinical and biochemical changes which cause aging have been linked to reduction in testosterone concentration. This approach is, however, too simplistic. Testosterone is the direct precursor to estradiol, which is the most potent endogenous estrogen, and to many other hormone derivatives which have a significant impact on the normal structure and on the function of the human body. Therefore therapy to increase testosterone is inextricably linked with estradiol supplementation. Here special attention should be paid to the fact that not all preparations of testosterone can be converted to estradiol and to other derivatives, which makes such supplementation seem not to improve, but, sometimes, to make the situation worse. Estrogen affects the skeletal bone and cardiovascular systems in many radical ways. The decline in estrogen is associated with osteoporosis, premature atherosclerosis, marked risk of myocardial infarction, and with loss of bone mass (Gooren \& Bunck, 2004).

While testosterone can act directly on cells, it can also be converted to dihydroxytestosterone (DHT) by 5 a-reductase. The same chemical reaction occurs which converts estradiol to 4hydroxyestradiol. In addition to hormonal effects, this compound has the peculiarity of saturation binding for dopamine receptors widely distributed in the brain. If one compares the chemical structure of dopamine with that of 4-hydroxyestradiol, it is possible to find the key to the dopamine receptor - a benzene ring with two hydroxyl groups attached to the ring which can be seen in Figure 8.

Dopamine receptors saturation in human brain has some very important implications of many physiological and pathological states. Dopamine deficiency causes Parkinson's 
disease. Dopamine plays a crucial role in our mental health. Also, male sexual fitness is closely related to hormonal balance nd especially to dopamine and 4-hydroxyestradiol. Outlined below are some of the entities associated with testosterone deficit or with the deficit of testosterone derivatives.

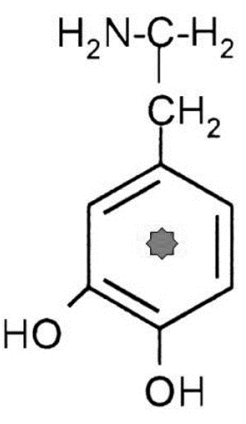

Dopamine

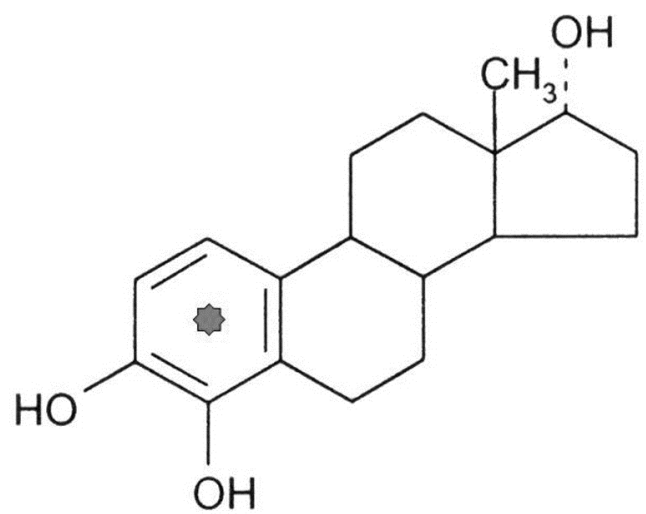

4-hydroxyestradiol

Fig. 8. The dopamine structure and the 4-hydroxyestradiol structure.

\subsection{Late - onset hypogonadism and type 2 diabetes}

Clinical trial results have shown that men with type 2 diabetes have a significantly greater testosterone deficiency (Zitzmann \& Nieschlag, 2006). In a large population study of subjects aged over 20, men with total testosterone levels in the lowest quartile and men with free testosterone in the lowest quartile had a 4 -fold higher prevalence of diabetes compared with men with testosterone levels in the first quartile $/ \mathrm{p}=0.04 /$ (Muller et al., 2005). Metabolic syndrome, defined as a combination of lipid disorders and cardiovascular risk factors: abdominal obesity, insulin resistance, and arterial hypertension, also increases the risk for late onset hypogonadism ((Kalyani \& Dobs, 2007). In my own research, out of 1200 men with late onset hypogonadism treated with hCG, more than 10 percent had diabetes. Raising the serum testosterone levels in these patients as a result of hCG therapy significantly improved glucose tolerance. Increasing testosterone levels, the average level of output being $18.3 \mathrm{nmol} / \mathrm{L}-38.6 \mathrm{nmol} / \mathrm{L}$, resulted in a reduction of $\mathrm{HbA1c}$ by an average of $1.75 \%$ $(p<0.001)$. This effect is shown graphically in Figure 9.

Normalization of HbA1c followed in the not-previously-treated diabetic group, as well as in the group of patients taking their medication without changing the dose. In some patients previously treated for diabetes, at the time of hCG administration, it was necessary to lower the dose or even to discontinue antidiabetic therapy. Among patients without known diabetes $(\mathrm{HbA} 1 \mathrm{c}<6.0 \%)$, as a result of hCG therapy, HbA1c reductions were observed by an average of $0.5 \%$. In patients with diabetes, insulin level was also determined. Average output level was $16.55 \mathrm{mU} / \mathrm{L}$, and with intensified hCG therapy the average calculated insulin level was $7.8 \mathrm{mU} / \mathrm{L}$.

I believe that the term "insulin resistance" is overused. Insulin resistance does not cause diabetes. The inability to metabolize glucose (burn sugar), fully and continually is the cause of type 2 diabetes. An increase in the concentration of the glucose in the extracellular space 
induces the increase in insulin levels. But insulin does not move sugar into cells when the cell-glucose level is equally high. Acceleration of tissue metabolism after injection of testosterone causes excessively fast burning of glucose, which decreases cell-glucose levels. Then, according to the concentration gradient, glucose enters the cell and this reduces extracellular cell concentrations of glucose. With the reduction of glucose concentration in the extracellular space, blood insulin levels fall below a certain level. Therefore "insulin resistance" does not apply in this case.

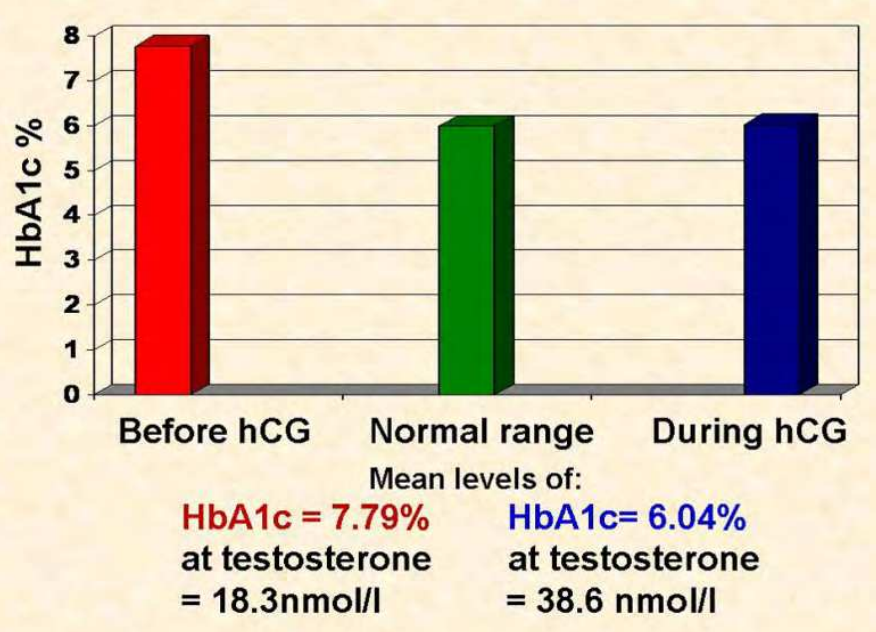

Fig. 9. HbA1c normalization as a result of hCG therapy.

\subsection{Late - onset hypogonadism and hyperlipidemia}

As men age, there is a dramatic decrease in testosterone production, with a corresponding decrease in the production of estradiol. Excess lipids in the blood lead to atherosclerosis. Cholesterol, which can be very annoying, is in fact absolutely necessary. Too high or too low a concentration thereof has a detrimental effect. LDL cholesterol serves as precursor for the synthesis of steroid hormones. Moreover, every cell in the body requires LDL cholesterol to maintain cell wall integrity. With age, cell turnover is reduced. What is more, there are lower cholesterol production rates to create new cells. An excessive reduction of cholesterol levels in the body results in the deficit of raw materials for cellular renewal and in the deficit in the synthesis of steroid hormones. Various formulations are present in the treatment for hypercholesterolemia. However, hypercholesterolemia can also be normalized only by accelerating the metabolic processes. Anabolic testosterone boost effectively corrects the moderate lipid metabolic disorders. The hCG hormone therapy, which results in a significant increase in testosterone concentration and in E2 concentration (in testosterone level - from $18.3 \mathrm{nmol} / \mathrm{L}$ to $38.6 \mathrm{nmol} / \mathrm{L}$; in E2 level - from $138.6 \mathrm{pmol} / \mathrm{L}$ to $280.9 \mathrm{pmol} / \mathrm{L}$ ), improves lipid profile. There is a 15 to $20 \%$ decrease in total cholesterol and its fractions. This effect is shown graphically in Figure 10. 


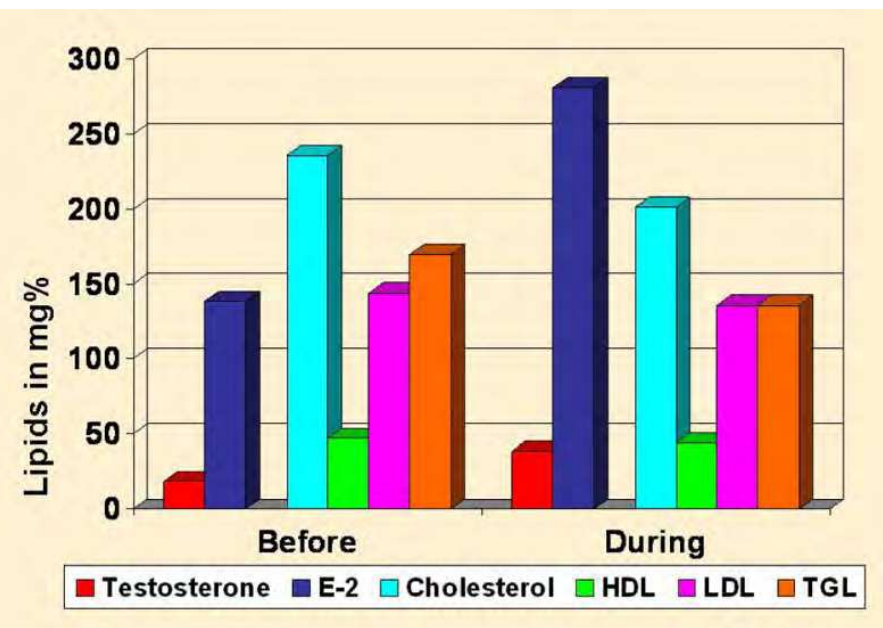

Fig. 10. Improved lipids during HCG-treatment hormonal treatment

\subsection{Late - onset hypogonadism and osteoporosis}

It is beyond discussion that women have osteoporosis as a direct result of ovarian hormone imbalance. In bone densitometry studies, $90 \%$ of those examined are females and only $10 \%$ are males; hence the great retardation of male endocrinology and of densitometric diagnosis of osteoporosis in men in relation to the same areas of medicine with regard to women. According to the recommendations of World Health Organisation, a woman should take hormone therapy early enough to avoid dangerous irreversible anatomical changes. What about a man? At puberty male estrogen can affect skeletal growth and bone mineralization. The study of men in the andropause also indicates a relation of estrogen level to bone mineral density (Riggs, et al. 2002). Studies have shown that two-thirds of the effects of testosterone replacement therapy (particularly with regard to increase in bone mineral) are due to excessive estrogen levels in aromatization of testosterone (Khosla, et al., 1998, Leder, et al., 2003). There is a clear relationship between the amount of androgens (bioavailable testosterone) and estrogens (bioavailable estradiol E2). In a large-scale study (data from 2623 men over 65, enjoying good health) levels of free and bioavailable testosterone and bioavailable estradiol levels correlated. Low levels of bioavailable estradiol were associated with age and with osteoporosis (Orwoll, et al. 2006). In our own panel of 1200 patients, it was found that raising man's testosterone levels to the values recommended for a given age was simultaneously accompanied by the increase of estradiol level. This caused the incorporation of calcium into bones, without any additional classical treatment of osteoporosis. The hormonal therapy used was to induce endogenous testosterone synthesis by human chorionic gonadotrophin (hCG). Please find below a depiction of the effects. A two year hCG therapy in a patient aged 60 years increased the bone density by $6.7 \%$ ( Figure 11 and Figure 12); and a long-term therapy could help restore bone density to that of a fine young man. Figure 13 shows densitometric data of the chapter's author (age 62y). Bone density of a fine young man - Young Ref $(\%)=92.7$; bone density with reference to age Age Match $(\%)=118.1$ Z-Score $=1.26)$ 


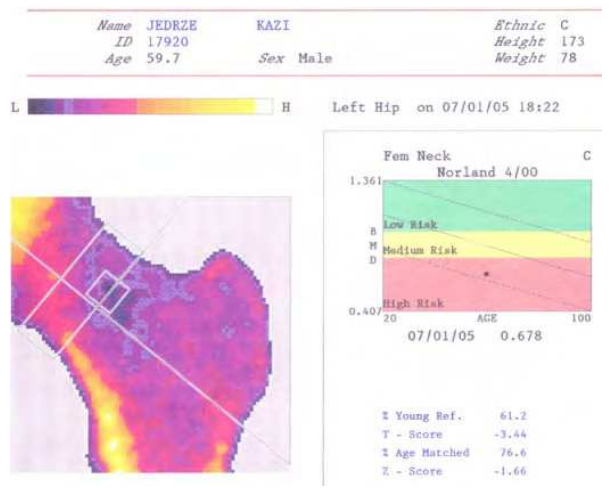

Fig. 11.

Fig. 12.

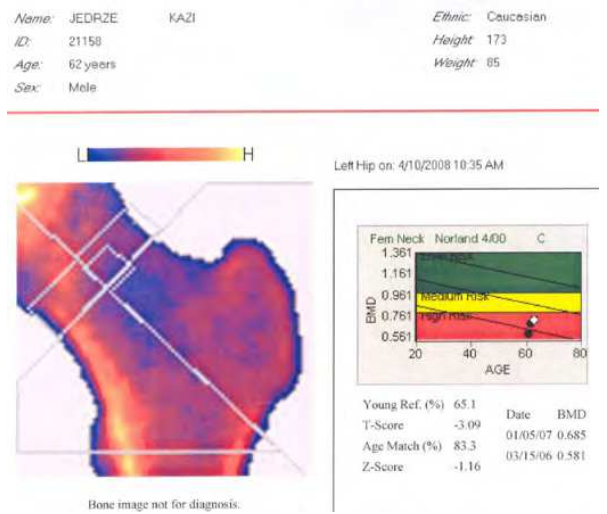

Name: GOMULA. ANDRZEJ

1D. 41607

Age: 62 years

Sex Male
Ethnic: Caucasian

Height 180

Weight 97

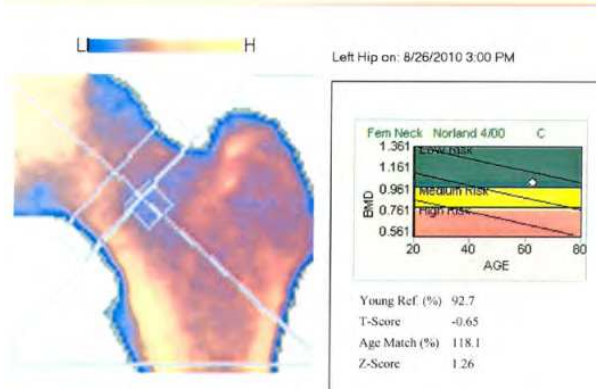

Fig. 13. 


\subsection{Late - onset hypogonadism, chronic prostatitis, BPH and prostate cancer}

It should come as no surprise to anyone that chronic prostatitis, benign prostatic hyperplasia $(\mathrm{BPH})$, and prostate cancer (PC) are consequences of hormonal deficits. Prostate-specific antigen (PSA), a marker that has been used for many years in early prostate cancer detection, turned out to be less than $100 \%$ accurate to diagnose prostate malignancy. PSA was originally supposed to be a unique marker for prostate cancer. It was supposed to be just what its name implies - a specific marker. Over the past few decades an enormous amount of material has been written about the role of PSA in diagnosis of prostate cancer. Doctors started to use other PSA values - such as PSA velocity, PSA density, and percentfree PSA - to get a more accurate idea of what was happening within the prostate. When the PSA reaches or exceeds the level of $0.5 \mathrm{ng} / \mathrm{mL}$, it is strongly recommended to take all-out diagnostic measures, in order to ascertain the absence of PC (Gould, et al., 2006). Practically in such cases, the biopsy of the prostate should be done. After a prostate biopsy, the PSA continues to rise, so the biopsy is repeated. This method often cannot give definite confirmation of prostate cancer, which has been the first dilemma. The other dilemma: what if the blood tests detect much lower levels of PSA? What if the reduction amounts to 2-3 fold? Long-term clinical experience shows that a sudden significant increase in PSA level is possible with any surgery, after virus infection regardless of its location, or with regard to a severe exacerbation of chronic inflammatory diseases. A sudden increase in one's PSA level should not escape the attention, but it is not equivalent to the occurrence or the development of prostate cancer. Changes in the PSA levels may be the consequence of the high levels of prostaglandins, which induce non-specific inflammations of the prostate usually seen in urine samples under a microscope.

Harbitz stated over 30 years ago that male gonadal dysfunction (endocrine testicular failure) leads to adenomas and to the prostate cancer (Harbitz \& Haugen, 1974). It is an undeniable fact that testosterone levels peak in a man at approximately age 30, but this does not yet cause $\mathrm{BPH}$ or prostate cancer. And the older the man, and the greater the chances of testosterone deficiency, the higher the prevalence of benign prostatic hyperplasia and of PC. It can no longer be doubted, questioned, whether $\mathrm{BPH}$ and PC are closely related to the hormonal changes in men, and practically to testosterone deficiency.

There are publications, which say that there was never any association between testosterone treatment, when subjects were all healthy men, and the increase of prostate volume and serum PSA level. They also say that in the treatment of men with hypogonadism, the therapy led to moderate prostate enlargement and to a $15 \%$ increase in PSA (Algarte-Genin, et al., 2004, Behre, et al., 1994, Gould \& Kirby, 2006). Among my own 1200 patients under treatment for hypogonadism, more than two-fold increase in endogenous testosterone concentrations after 37 months of hCG therapy resulted in a $40 \%$ decrease in their PSA levels. The aforementioned decrease is closely related not only to concentration of testosterone, but to the level of estradiol, which stimulates LHRH mRNA synthesis and increases pituitary LH synthesis. Testosterone deficiency does not cause prostate pathology. However, the losses of testosterone and long-term E2 deficiency result in the situation in which the hypothalamus and pituitary gland can become prostate's greatest enemies. Therefore, modern hormonal medications used to treat BPH have selfstimulation points in the lateral hypothalamus (Oesterling, 1991, Reissmann, et al., 2000, Debruyne, et al., 2008). 


\subsubsection{Late - onset hypogonadism, BPH and chronic prostatitis}

The symptoms of $\mathrm{BPH}$ are practically only recognized when there are irreversible anatomical changes in the prostate, causing LUTS. Patients with BPH often see the doctor when they already have symptoms of LUTS. Meanwhile, the symptoms of chronic prostatitis will be in place long before that. Antibiotic or other medications are rather ineffective in those patients and the hormone therapy can help to relieve clinical symptoms. The effects of hormonal disbalance, including testosterone deficiency, can be seen on transabdominal and transrectal ultrasound scans.

If hypoechoic areas in the transition zone of the prostate (marked with dots in Figures $14 \div 17$ ) are noted in ultrasound, it is a common sign of deficient testosterone levels. Such changes are occasionally described as inflammation and are not paid due attention to.

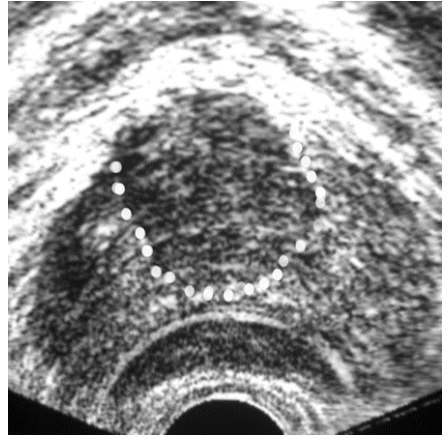

Fig.14.

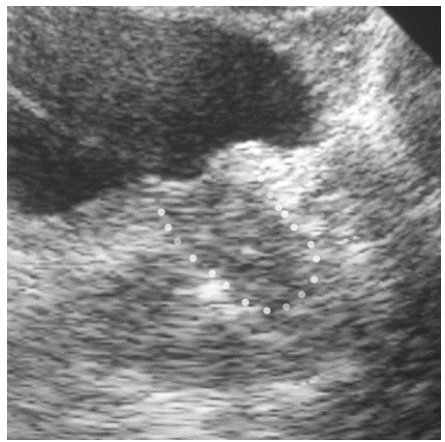

Fig. 16.

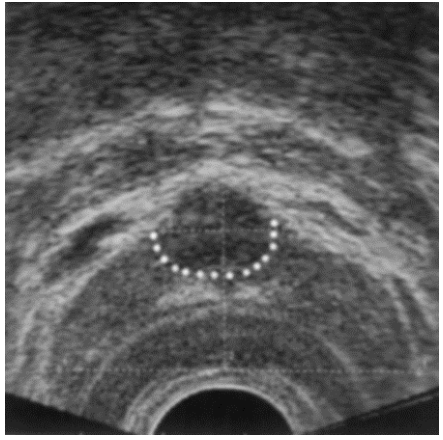

Fig. 15.

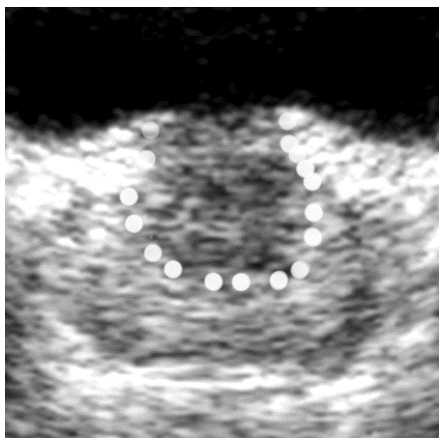

Fig. 17.

Figure 14. $\div$ 17. Ultrasound scan images of the prostate of testosterone deficiency patients. Figure 14 \& 15. Transrectal ultrasonography. Figure 16 \& 17. Abdominal examination. Hypoechoic area marked by dots.

In my 30 years of being an urologist, I have treated hundreds of men diagnosed with prostatitis by other physicians. Antibiotics treatment could last several weeks or even months. Worst of all, it was not effective. Treatment with hCG led to the freedom from symptoms within 1-3 weeks. These symptoms were associated with the spread of prostate adenoma which, as it can lift the bladder, causes prostate inflammation symptoms. During hCG therapy, there is a reduction in adenoma, as demonstrated by MRI (Figures $18 \div 20$ ). 


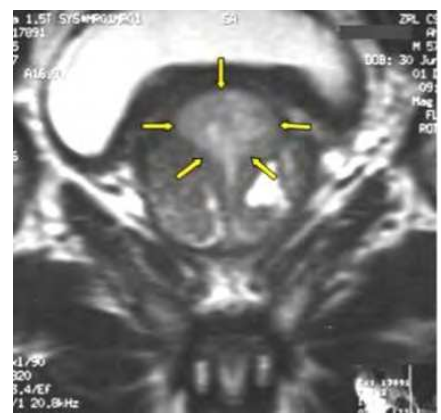

Fig. 18.

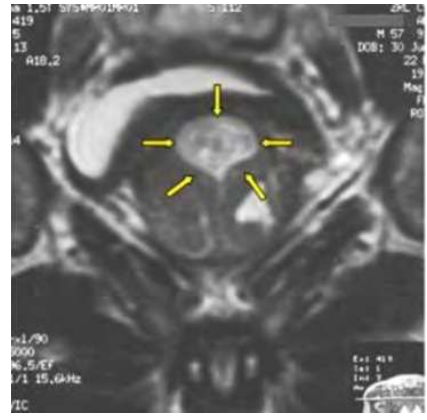

Fig. 19.

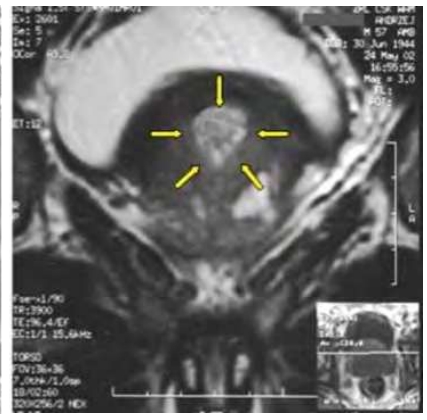

Fig. 20.

Fig. 18. - 20. Prostate MRI before hCG therapy (Fig. 18), after 3 months of hCG therapy (Fig. 19), and after 6 months of hCG therapy (Fig. 20). Arrows indicate regions of prostate adenoma - hyperplasia of the transition zone - which as a result of hCG treatment was markedly reduced.

Some experts now recommend alpha-blockers and hormones as first-line treatment for patients with moderate to severe BPH symptoms. It must be emphatically stated that alphablockers therapy appears to have no effect on the disease; it does not hinder its development; it only mitigates symptoms of $\mathrm{BPH}$ and facilitates urination. But after using alpha-blockers for a number of years adenoma can enlarge to the extent that surgical treatment becomes necessary. At the same time, there will be irreversible pathological changes of the bladder wall, which disrupt the normal function thereof. Thus, alphablockers do not solve the problem of treatment for BPH.

In hormonal therapy of BPH the agents used are: 1.- 5a-reductase inhibitors, and 2. - drugs, which inhibit LH synthesis or LHRH synthesis.

Ad. 1. 5-alpha reductase inhibitors block the conversion of testosterone to dihydroxytestosterone. Their effectiveness in the management of BPH is debatable. Laurie Barclay, MD (Pfizer), issued a statement used in Pfizer commercial that Finasteride was no better than placebo in treating BPH symptoms. In fact, 5 alpha reductase inhibitors may cause hot flashes, decreased libido and impotence (Oesterling, 1991).

In my opinion, this drug works, but its metabolic and functional consequences mean that the balance of potential profit versus loss can be harmful for men. Therefore I have not been using 5 alpha reductase inhibitors in the treatment of $\mathrm{BPH}$.

Ad. 2. In the treatment of BPH, other drugs have been used as well to decrease LH synthesis for more than 20 years. This effect has been achieved by LHRH analogues or LHRH agonists (Oesterling, 1991, Reissmann, et al., 2000, Debruyne, et al., 2008). This therapy, however, has significant disadvantages. It blocks testosterone endosynthesis, which leads to metabolic and functional complications. A fundamental question arises: What kind of man will deprive himself of potency and disrupt his normal body chemistry and metabolism in order to improve the health of his prostate? And so, shall we provide such a treatment, if it can block LH synthesis, and, while at the same time, more than a twofold increase in testosterone undoubtedly can positively affect the biology of man?

\subsubsection{LOH and prostate cancer}

Half a century ago arose the bizarre notion that testosterone was really dangerous and that it could cause prostate cancer. Years later, it became clear that there was no credible 
scientific basis for the claim that high levels of testosterone accelerated development of prostate cancer. In fact, the opposite is true (Bonczyk, et al., 2008). The theory that testosterone may stimulate the growth of prostate cancer originates from 1941 when Huggins and Hodges reported that carcinoma was androgen-dependent. They demonstrated the importance of lowering testosterone levels in prostate cancer progression. Huggins and Hodges won the 1966 Nobel Peace Prize for their groundbreaking work revealing that castration causes regression of prostate cancer; while testosterone may cause its progression (Huggins \& Hodges, 1941). It is now known that a multiplicity of data contradict Huggins and Hodges's contention that testosterone accelerates prostate cancer growth. Apparently it was negligent misinterpretation of one patient observation (Morgentaler, 2006). And they won the Nobel Prize. This caused a distorted look at the testosterone-prostate cancer link over the last half century. Even 15 years ago in Poland, testosterone was considered a carcinogen by Polish Ministry of Health and Social Welfare (Official Law Daily, 1996). There is no evidence that among men with prostate cancer, serum testosterone concentration is higher than in the rest of the population. On the other hand, low testosterone values coexisting with prostate cancer are associated with worse prognosis, a lower degree of differentiation, and a higher degree of severity of cancer (Morgentaler, 2006, Yano, et al., 2007). Moreover, there is no convincing evidence showing the effect of testosterone on the development of pre-cancerous prostate condition. Rhoden et al. determined that the risk of the development of prostate cancer in hypogonadal patients receiving testosterone, with and without accompanying HG-PIN, does not increase with hormone therapy. After twelve months, the risk was 1,2\% in men without HG-PIN, and 5\% in men with HGPIN detected. The aforementioned values do not exceed those in the general population (Rhoden \& Morgentaler, 2003). At the same time, one can find statements that there are two classic contraindications for the administration of testosterone, namely suspected or histologically proven prostate cancer and symptomatic benign prostatic hyperplasia (Montorsi, 2007). While I understand precautions that most men with prostate cancer should avoid testosterone therapy, even though I use it for my patients, a total ban on the use of testosterone to treat BPH appears to be an anachronism.

25 years ago, Fowler and Whitmore proposed the concept of "saturation" in order to explain the relationship between prostate cancer and serum testosterone levels. It explains why the increase in serum testosterone levels in patients treated with that hormone does not cause the disease (Fowler \& Whitmore, 1981). The correctness of this concept is confirmed by observations of patients, despite treatment with $\mathrm{T}$, there was no increase in PSA or prostate volume. In men suffering from hypogonadism the treatment did not lead to excessive growth in prostate size, and it led to an only 15\% increase in PSA (Algarte-Genin, et al., 2004, Gould \& Kirby, 2006). Currently, it is believed that exogenous testosterone treatment in patients with PC, which does not follow androgen ablation, should not worsen prognosis of cancer (Bonczyk, et al., 2008). It has long been feared that the Late Onset Hypogonadism $(\mathrm{LOH})$ treatment by administering testosterone may increase the risk of high-grade prostate cancer. Numerous studies show that there is no PSA increase during testosterone therapy. Among my own 1200 patients under treatment for hypogonadism, more than two-fold increase in endogenous testosterone concentrations after 37 months of hCG therapy resulted in a $40 \%$ decrease in their PSA levels. In each age group - and the older the man, the more severe it is - there are patients who present insufficient endosynthesis, but they constitute only a few percent of total number of patients. During the extended follow-up of these patients, it has been found that $14 \%$ of 
them have had some form of prostate cancer within 3 to 36 months. The results clearly show that there is a greater decrease in endogenous testosterone synthesis, but at the same time there is an increased risk of prostate cancer. The patients in question had very high LH/PSA ratio. Therefore prostate cancer is not caused by testosterone, but, to the contrary, it is a consequence of the development of testosterone deficiency. Moreover, there is a correlation between testosterone levels and the degree of PC malignancy (Hoffman, et al., 2000). It has been known for years that patients diagnosed with prostate cancer and with high Gleason scores, have lower than normal levels of testosterone. Lower testosterone levels result in greater PC malignancy (Schatzl, et al., 2001). Patients with high Gleason score prostate cancer have lower testosterone and estradiol serum levels.

There is also no evidence that men with prostate cancer have a much higher testosterone concentration than the normal population. On the other hand, low testosterone values found in men with prostate cancer are associated with poorer prognosis, with a lower degree of differentiation of PC, and with a higher degree of PC severity (Hoffman, et al., 2000, Morgentaler, 2006, Yano, et al., 2007). In men treated with testosterone, prostate cancer was detected in biopsies of $1 \%$ of patients, while in men with the prevalence of hypogonadism in $14.3 \%$. There are some reports that testosterone therapy may reduce the risk of prostate cancer (Fowler \& Whitmore, 1981, Prout \& Brewer, 1967). Only some reports, because who would dare to publish anything incompatible with the study by Nobel laureates?

\subsection{Late - onset hypogonadism, sexual drive, potency and libido}

For many years, we have been reassured that only androgens have a key role in both stimulating and maintaining sexual function in men. It was believed that testosterone and the existence of a normal level of libido were inseparably connected (Shabsigh, 2003, Morales, et al., 2004). Nevertheless, sexual dysfunction in men is directly associated not only with testosterone but also with estradiol (E-2) and with other neurohormonal factors. Erectile dysfunction was linked to the development of benign prostatic hyperplasia (BPH), without even taking into account the fact that both benign prostatic hyperplasia (BPH) and erectile dysfunction (ED) were often caused by the hormone deficit. The effects of women's hormone replacement therapy on their psychological and sexual functioning are still the subject of the research in the borderline field between medicine and psychology. Female hormone therapy in treating menopause is common around the world. Meanwhile, several years ago it was stated that the effects of this therapy on men were not known. Hormone replacement therapy for men has been lagging for at least 20 years compared with hormone replacement therapy in women (Tenover, 1999). There is a decline in testosterone production in elderly men that can lead to a decrease in sexual desire (Kaufman \& T'Sjoen, 2002). Androgen therapy can stop and even reverse this degenerating process (Hajjar, et al., 1997, Morales, et al., 2004). Information on the importance of testosterone in male sexuality is often divergent. It is known that surgical and pharmacological castration leads to impotence. On the other hand, it is recognized that testosterone deficiency is considered to be of little importance in the development of erectile dysfunction or in life force. (Anderson, 2003, Montorsi et al., 2003).

There was no sexual orientation change in men who underwent surgical castration. Sexual desire was preserved by the majority of the patients, but interest in sex decreased, which was associated with decreased frequency/intensity of orgasms (Zverina, et al., 1990). Testosterone replacement therapy suitable for men with primary testicular failure as a result 
of surgery conduces to a return to good sexual health and to good psycho-social outcomes (Fossa, et al., 1999). A drop in men's testosterone levels results in reduced libido and sexual potency. The implementation of hormonal therapy in hipogonadal men at the time when testosterone levels increase significantly, approaching the upper limit for normal, causes regular nocturnal erections. It also increases the number of spontaneous erections and it increases sexual activity (Burries, et al., 1992, Tariq, 2002). Other authors also propose that libido is closely tied to testosterone levels and that hormone replacement therapy increases the frequency of sexual thoughts and significantly improves one's libido (Davidson, et al., 1982, Kwan, et al., 1983). Testosterone induces nitric oxide synthesis in vascular endothelium through its influence on arginase activity. This leads to the opening of vascular pathways; facilitates blood flow into the corpora cavernosa; and enhances penile erection. The same mechanism is used in treatment of erectile dysfunction (ED) with PDE-5 inhibitors. It is currently the primary means of treating ED.

However, such treatment might not be effective when there is a decrease in male libido (the decrease being one of the symptoms of andropause) as a result of a decrease in the level of testosterone which is a hormone produced in a man's testicles. The occurrence of erectile dysfunction causes an increase in depression. Meanwhile, the treatment of depression through the introduction of SSRIs (selective serotonin reuptake inhibitors) affects the deterioration of erectile function in a secondary way (Hsu \& Shen, 1995, Keller, et al., 1997). Thus, Andropause Erectile Dysfunction Treatment and male depression treatment are often ineffective. It is worth remembering, however, that andropause can start from any age but generally around age 30, a relatively young age, when a man still has some 40 years of life. Erectile dysfunction, especially in young men, has not yet been linked with their hormonal status.

I have found that during hormone therapy my patients are affected by changes in their sexual health. A thorough analysis of the problem became the subject of the doctoral dissertation of one of my assistants (Czyżowska, 2009).

My research on 88 men aged 20-68 years, (mean age 45), clearly shows an increase in testosterone endosynthesis at the time of hCG Hormone Therapy, on average from 17.93 $\mathrm{nmil} / \mathrm{L}$ to $40.86 \mathrm{nmol} / \mathrm{L}$. At the same time, a significant increase in E-2 was found in those examined, on average from $168.72 \mathrm{pmol} / \mathrm{L}$ to the value of $332.44 \mathrm{pmol} / \mathrm{L}$. The change in hormone levels of those examined resulted in a significant improvement in their sexual performance, in libido and in erectile potency, evaluated using the International Index of Erectile Function (IIEF-5) questionnaire. The average value of IIEF-5 before therapy was 13.4 points $(\mathrm{SD}=5.0)$, and during therapy -19.88 points $(\mathrm{SD}=4.3)$. The data are shown in numbers in Table 7 , and graphically in Figures $21 \div 22$.

\begin{tabular}{|c|c|c|c|}
\hline & $\begin{array}{c}\text { Before therapy } \\
\text { (average, SD) }\end{array}$ & $\begin{array}{c}\text { During therapy } \\
\text { (average, SD) }\end{array}$ & Significance \\
\hline Testosterone & $17.937 \mathrm{nmol} / \mathrm{L}, 7.11$ & $40.89 \mathrm{nmol} / \mathrm{L}, 12.55$ & $\mathrm{p}<0.0001$ \\
\hline Estradiol & $168.72 \mathrm{pmol} / \mathrm{L}, 77.9$ & $332.44 \mathrm{pmol} / \mathrm{L}, 141.72$ & $\mathrm{p}<0.0001$ \\
\hline IIEF-5 & $13.4,5.0$ & $19.876,4.3$ & $\mathrm{p}<0.001$ \\
\hline
\end{tabular}

Table 7. Average hormone concentrations and the IIEF-5 score before and during treatment 


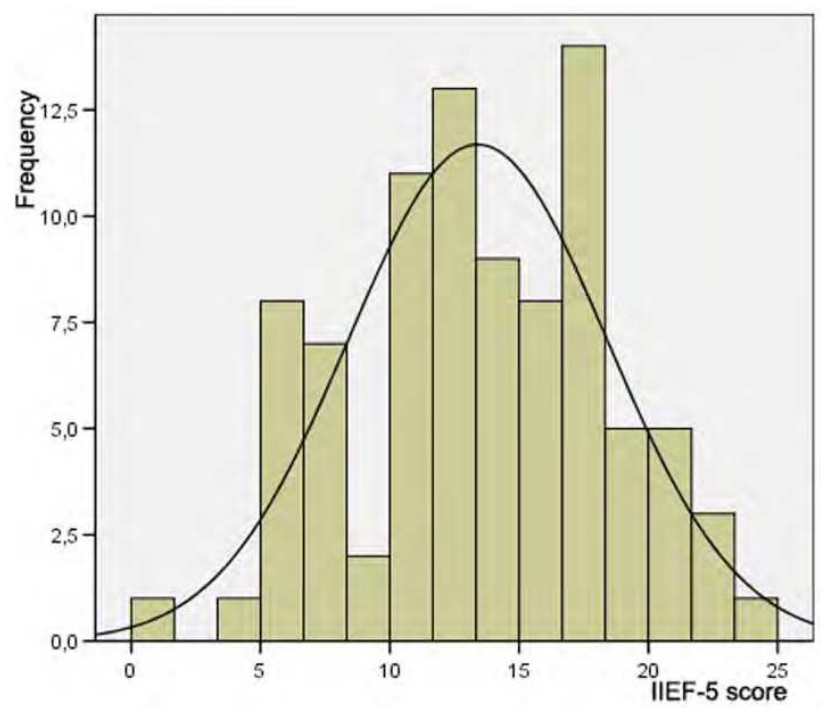

Fig. 21. Average IIEF-5 score before treatment

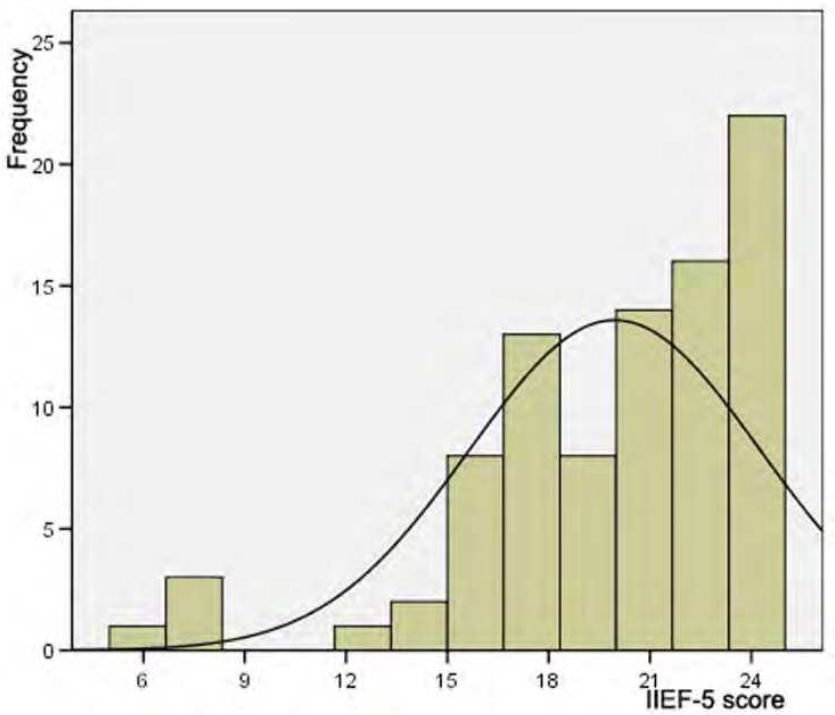

Fig. 22. Average IIEF-5 score during treatment

According to the Arizona Sexual Experiences Scale (ASEX), there was also a significant improvement in sexual health, from an average of 17.39 points $(S D=4.24)$ before treatment to 12.45 points $(S D=3.57)$. The average ASEX values distribution has been shown in Table 8. 


\begin{tabular}{|c|c|c|c|c|c|c|c|c|c|c|}
\hline & \multicolumn{3}{|c|}{$\begin{array}{c}\text { Before therapy } \\
\text { (average, SD) }\end{array}$} & \multicolumn{3}{c|}{$\begin{array}{c}\text { During therapy } \\
\text { (average, SD) }\end{array}$} \\
\hline Testosterone & \multicolumn{3}{|c|}{$17.937 \mathrm{nmol} / \mathrm{L}, 7.11$} & \multicolumn{5}{c|}{$40.89 \mathrm{nmol} / \mathrm{L}, 12.55$} \\
\hline Estradiol & \multicolumn{3}{|c|}{$168.72 \mathrm{pmol} / \mathrm{L}, 77.9$} & \multicolumn{5}{c|}{$332.44 \mathrm{pmol} / \mathrm{L}, 141.72$} \\
\hline Scale - ASEX & 3.9 & 3.4 & 3.6 & 3.1 & 3.3 & 2.6 & 2.3 & 2.6 & 2.5 & 2.5 \\
\hline
\end{tabular}

Table 8. Average hormone concentrations and the Arizona Sexual Experiences Scale measurement before and during therapy.

As noted above, serum concentration of E-2 plays a dominant role in male sexual health. Low levels of aromatase, which result in extremely low E-2 concentrations, may, nevertheless, be important in male sexual performance without affecting sexual orientation and gender identity (Gomuła, 2006, Gomuła, 2007). In patients with congenital absence of aromatase only the low-dose E-2 substitution results in significant changes in sexual behavior. Estradiol administration leads to increased erotic fantasies, masturbation or sexual activity Carani, et al., 1999).

My own research clearly shows that E-2 is required to maintain sexual functions in adult men (Gomuła, 2007). The manifestations thereof have been observed after prolonged hormonal therapy. Androgen deficiency patients had their testosterone levels increased for therapeutic purposes. During the therapy, a parallel increase occurred in serum E-2 concentrations, as the effect of the all natural aromatase. Some patients had such high E-2 levels that they exceeded the normal physiological range. In order to reduce E-2 concentration, my patients received preparations blocking aromatase activity. As a result of this therapy, men characterized by high concentrations of testosterone (falling in the upper limits of normal), whose E-2 was detected at very low levels, had a total loss of libido. At the same time those men suffered from erectile dysfunction, which could even lead to inability to initiate or maintain an erection. Stopping the drug which blocked aromatase resolved the symptoms and resulted in a rapid return of high concentrations of E-2. Some authors report that in the activation of male sexual behavior the brain level conversion of testosterone to estradiol is of major importance and that testosterone's effects are not in themselves so important (Balthazar \& Ball, 1998). Testosterone has a significant effect on the smooth muscle in the corpora cavernosa. Androgens may significantly affect the ultrastructure of the corpora cavernosa and these changes are responsible for erectile dysfunction (Traish \& Kim, 2005).

In young men, the ratio between smooth muscle and stroma in the corpus cavernosum is 1:1. Long-term hypogonadism causes the ratio of contents in muscle tissue to be 1:5, which results in smooth muscle atrophy and in fibrotic changes of the corpora cavernosa (Yassin \& Treish, 2004).

Fig. 23 represents normal cavernosal histological appearance and its loss as a result of testosterone deficiency, according to Yassin (Yassin \& Treish, 2004).

Androgen replacement can lead especially in hypogonadism patients to recovery process within the trabecular tissue. This result means that testosterone therapy supports the "recovery process" not only in striated muscles in human body / reversible process/ (Yassin \& Treish, 2004).

The basic question arises as to what should the first step be in a successful erectile dysfunction prevention and in a successful erectile dysfunction treatment? If the decrease in testosterone leads to penile tissue fibrosis, smooth muscle atrophy in corpora cavernosa, and 
damage to mechanisms blocking the blood flow from the corpora cavernosa, how do PDE-5 inhibitors pour the proverbial oil on the troubled waters? And at the same time testosterone restores penile smooth muscle.
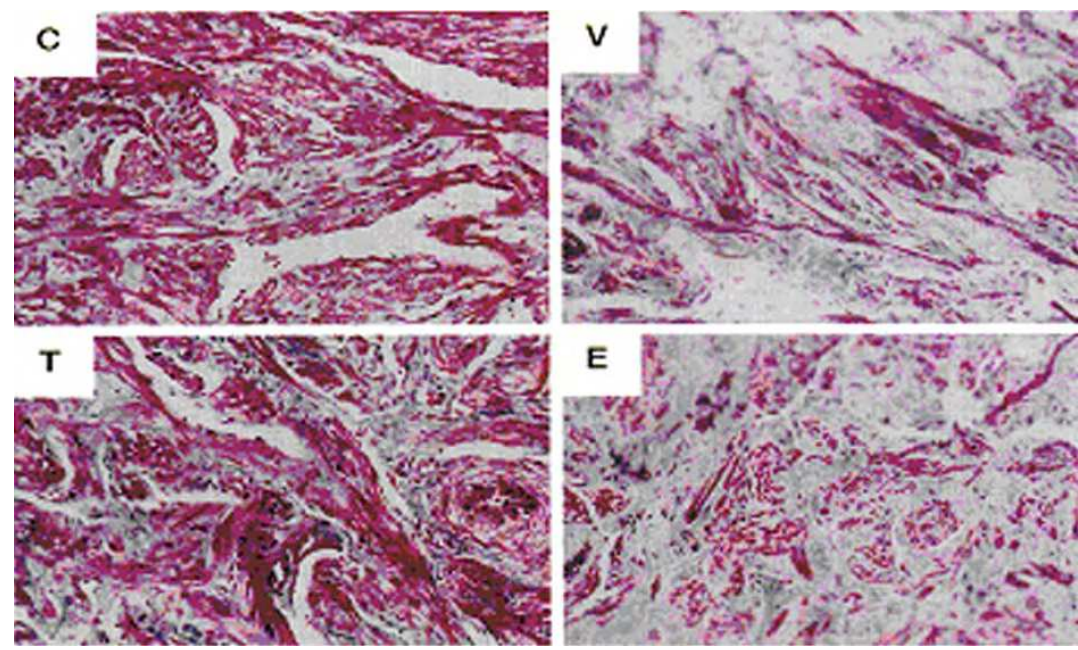

Fig. 23. Aging changes in the cavernosal tissue (Fig. 23-V) can also be induced by androgen deprivation (Fig. 23-E). The ratio of smooth muscle compartment to connective tissue which is normally 1:1 (Fig. $23 \mathrm{C} \& \mathrm{~T}$ ) would suffer a shifting till 1:5 (Fig. $23 \mathrm{~V} \& \mathrm{E}$ ) with a higher mRNA concentration as an expression of increasing number of alpha adrenoceptors according to Yassin (Yassin \& Treish, 2004).

Testosterone deficiency is closely linked to the reduction in libido. And when one's libido is lowered, the effectiveness of PDE-5 inhibitors is also limited. In such situations, the preventive and curative procedure is to maintain adequate testosterone level in the body. What is more, in patients receiving PDE-5 inhibitors, the increase in the concentrations of testosterone can improve the penile vascular blood flow, which is yet another argument for using the aforementioned procedure.

It is also known that diabetes can increase the problem of erectile dysfunction. It was previously believed that diabetes lead to lower testosterone levels. In contrast, quite the opposite is happening. A drop in testosterone levels due to a decrease in glucose uptake facilitated by anabolism reduction causes diabetes. Increased levels of testosterone significantly improve glucose tolerance and reduce one's insulin levels and the HbA1c level. Testosterone deficit can thus affect sexuality, both at a particular moment and through changes in metabolic processes leading to vascular lesions. There may also be a significant delay in the increase/drop in testosterone, even for many years, which may lead to erectile dysfunction (Gomuła, 2006). Some argue that the very fact of the occurrence of erectile dysfunction, rather than the testosterone concentration level, is the main indication for hormone replacement (Shabsigh, 2003). But the mechanism of erection is based not only on testosterone levels. It is not based merely on the E-2 either. Because neither testosterone nor E-2 alone determine the adequacy of an erection. If the mechanisms of erection depended on changes in serum testosterone levels, one would 
have to wait many hours for an erection. Testosterone concentrations and E2 concentration increase as a result of natural endosyntesis. This lasts about 6 hours because that much time elapses from the original signals for spectral contrast in visual cortex during the first stage of sleep, rapid eye movement (REM). And the highest concentration of testosterone for a man is at approx. 4-5 am, after about six hours of sleep. The mechanisms of erection depend on such factors as the concentration of hormones, but they do not depend on them in a direct way. To obtain or maintain an adequate erection, one needs adequate levels of testosterone and of E-2. Therefore, aromatase, which converts testosterone to E-2, is also essential. Arginase, which induces the synthesis of nitric oxide in vascular endothelial cells, also plays an important role. 4-hydroxyestradiol derivative of estradiol is a substance that at the level of brain activity plays perhaps the most important role therein. 4-hydroxyestradiol has this property that it may saturate dopamine receptors in the brain. At high saturation level, dopamine stimulates these receptors, causing penile erection. Release of dopamine, which is one of many neurotransmitters, occurs rapidly. These two mechanisms are shown in Figure 24.

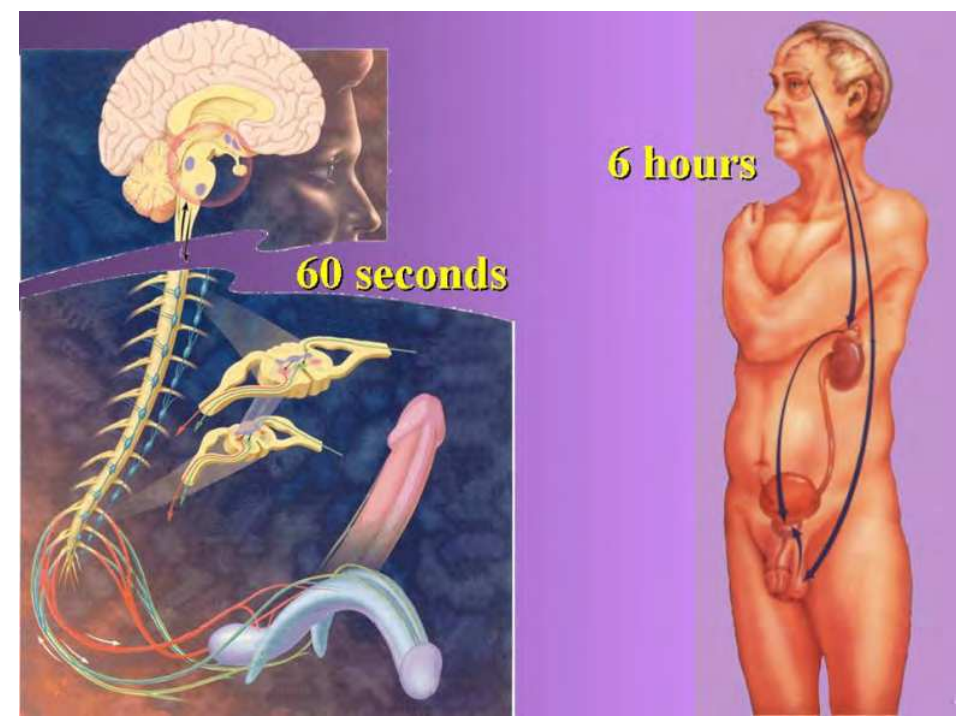

Fig. 24. Erection occurs as a result of the involvement of neurotransmiters, secreted at the time counted in seconds, and does not depend on hormonal changes, which can last for hours.

It clearly shows that we need to revise our views on the impact of hormones on our sexuality, potency, and libido, because not just hormones but also brain neurotransmitters have an impact on male sexuality.

\subsection{Late - onset hypogonadism and depression}

I have observed in my patients a change in their mental condition while they were under hormone therapy. A state of depression transformed itself into a state of joy. This gave 
enough reason to conduct a thorough analysis of the problem, which turned out to be the topic of the PhD dissertation of one of my assistants (Czyżowska, 2009).

Depression is a serious medical condition where a person may feel "down" or "hopeless" for weeks or more. According to the National Institute of Mental Health, the signs and symptoms of depression include: persistent sad, anxious, or "empty" mood; feelings of hopelessness, pessimism; feelings of guilt, worthlessness, helplessness; loss of interest or pleasure in hobbies and activities that were once enjoyed, including sex; decreased energy, fatigue, being "slowed down"; difficulty concentrating, remembering, making decisions; insomnia, early-morning awakening, or oversleeping; appetite and/or weight loss, or overeating and weight gain; thoughts of death or suicide, suicide attempts; restlessness, irritability; persistent physical symptoms that do not respond to treatment, such as headaches, digestive disorders, and chronic pain. Treatment of sexual disorders caused by depression with antidepressants based on Selective Serotonin Reuptake Inhibitor (SSRI) has a secondary detrimental effect on erection (Hsu \& Shen, 1995, Keller, et al., 1997). It results in a vicious circle - antidepressants increase erection disorders, while sexual life disorders intensify depression. The circle is closed. How can it be broken?

Evidence proving therapeutical effect of estradiol in depression disorders has been found amongst women, however the research has not proven explicit relation between the estradiol level and depression among men (Studd \& Panay, 2004). Neurosteroids produced in the central nervous system from cholesterol or other steroidal precursors are responsible for direct functioning within the brain, while alterations in their production typical for menopause in women and men cause certain aging symptoms. They directly influence, for example, body temperature regulation (hence the heat waves and cold sweats symptoms), memory function and emotions. Situational factors experienced by the given person, such as stress, secondarily disturb their secretions of hormones (Carruthers, 2004).

Research results demonstrated that hormone therapy for men, with hCG inducing testosterone endosynthesis causing over a double increase of testosterone and E-2 concentrations, result in substantial improvement of psychological condition and not only relieve depressive states, but also causes a progression from depression to the state of joy of life.

Based on the author's own research on 88 men aged between 20 and 68 years, (mean age 45 years), during the hormone therapy with hCG testosterone endosynthesis increase was achieved from E-2 average value $168.72 \mathrm{pmol} / \mathrm{L}$ up to $332.44 \mathrm{pmol} / \mathrm{L}$. The change of hormonal state of the subjects resulted in substantial improvement of their psychological state assessed on the basis of extended Beck Depression Inventory /BDI/ (Beck, 1967).

The classic BDI evaluates exclusively depressive state. Condition above zero is not assessed, only depression is included. Accepting BDI as a good test however not designed to evaluate joy of life state, I have developed the questionnaire adding reflection towards opposite direction, i.e. towards joy of life. Implementing such a tool for analysis of patients' psychological state enabled the change of hormonal condition to be demonstrated as not only what causes relief from depression, but also progress to the state of joy of life.

Restoring man's normal concentration of testosterone and E-2 results in significant improvement of his mental health; depressive states subside. Furthermore, patients who prior to treatments were below state " 0 ", in depression, during the therapy note states significantly above state " 0 "; they move to the joy of life state, as presented in Fig. 25. 


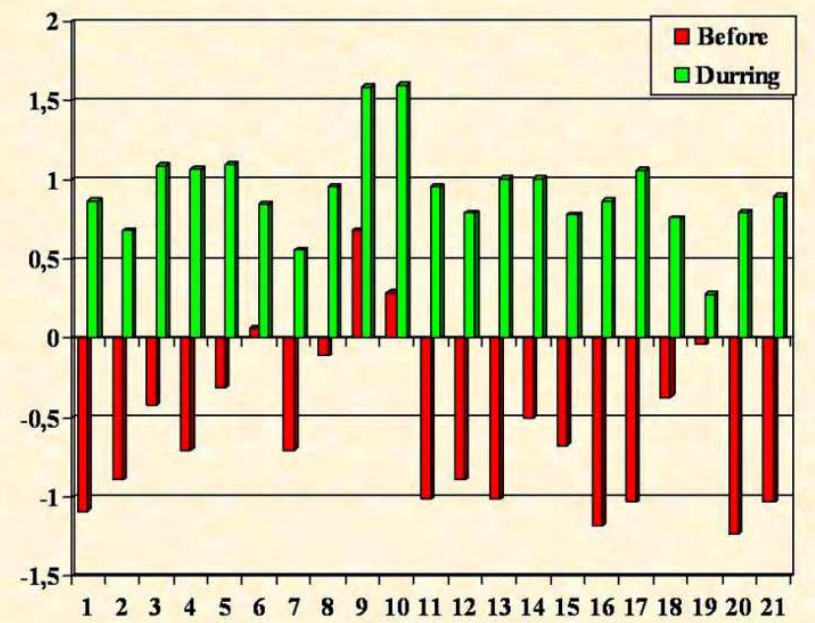

Fig. 25. Psychological state according to BDI: before the therapy - red, after the therapy green. Patients who suffered from depression are in the joy of life area during the treatment.

The facts above presented explicitly demonstrate a close correlation between hormonal and psychological states of man.

\subsection{Late - onset hypogonadism and Parkinson's disease}

Although Parkinson's disease (PD) is diagnosed in only $0.1 \%$ of the total population, in the population older than 70 years of age its prevalence reaches $1 \%$. The mean age of developing the disease is 58 years, and PD affects men more frequently than women /1.6:1/ (Edwards, et al., 2002, Nelson, 2002). The main cause of PD is the degeneration of dopaminergic neurons of substantia nigra in the nigro-striatal system of the brain. The development of the disease is believed to be triggered by both genetic and environmental factors. So far there have been very few reports in the literature, linking PD with the hormonal condition of a patient. Yet numerous symptoms resulting from the deficiency of such sex hormones as testosterone in men and progesterone-estrogen group in women, may co-exist with motor and other PD symptoms. These include impotence, lowered joy of life, lack of energy and mental depression (Edwards, et al., 2002, Nelson, 2002). Experimental studies have shown that estrogens can protect hippocampus neurons against beta-amyloid protein, which is implicated in the development of PD. The estrogens influence a creation of new synapses and improve the survival of neurons in hippocampus region (Serwin, 1994, Okun, et al., 2001).

Recent studies suggest the existence of morphogenic influence of estrogens on neurons and on plasticity of synapses. Estrogens appear to provoke synapsogenesis in hypothalamic cells. Apart from that, nonspecific systems activating brain neuroplasticity processes seem to be modulated as well (Skibińska \& Kossuth, 2003).

The neuroprotective action of estradiol is connected with oxidation processes. Oxidative stress is known to be important in the process of dopaminergic neuronal degeneration in Parkinson's disease, whereas estrogens have neuroprotective effects in neurodegenerative 
disorders (Sawada, et al., 1998). HRT in men has been used only recently and never so widely as in women, thus we have less experience for HRT in men.

Regardless of the fact that the relation between dopamine and androgens at the level of the central nervous system is still not absolutely clear, on the basis of my own clinical observations it can be stated categorically that in men there exists a strong correlation between the hormonal system and PD symptoms. This statement is supported by the initial examinations and observation of PD patients who consider to undergo HRT.

The preliminary study comprised of 16 men suffering from PD and LOH, aged 64 -78 years. The hCG therapy used was to induce testosterone endosynthesis. The follow-up lasted from 1 to 4 years.

Following the therapy, the serum testosterone concentration increased from the mean baseline level of $12.3 \mathrm{nmol} / 1$ to $34.8 \mathrm{nmol} / \mathrm{L}$. So far, the main parameter that has been considered for HRT in men has been the testosterone concentration. When the hCG was administered, though, the testosterone concentration increase was accompanied by simultaneous increase of estradiol level. In the analysed group of patients, the increase of the mean estradiol level was from the baseline of $46 \mathrm{pmol} / \mathrm{L}$ to $189 \mathrm{pmol} / \mathrm{L}$.

The addition of the hormonal therapy was supplemental to the standard long-term pharmacological anti-PD treatment. As a result of this addition, a dramatic improvement of PD symptoms was observed.

Before the hormonal therapy began, the patients complained of motor latency and insufficiency, hand tremors, muscular tremor and rigidity in legs. During the treatment these symptoms clearly improved or completely disappeared.

Just stating the fact, though, without being able to document it, may justly be questionable. Numerical assessment and analysis of parameters from various questionnaires are ambiguous. That is why every possible effort has been made to present undeniable proof and evidence of beneficial changes in the clinical condition of PD patients. The first evidence for the relationship between $\mathrm{LOH}$ and clinical symptoms of PD is the film which was shown during ISSAM Seminar 'Androgen Deficiency in the Adult Male' at the $4^{\text {th }}$ World Congress of The Aging Male in Prague.

Dr Malcolm Carruthers, a Congress participant, wrote in his book, published a few months after the Congress: "The results showed that after treatment, Parkinsonian symptoms greatly improved. Physical symptoms of slow, limited movement (bradykinesia), hand tremor, muscular tremor and rigidity of the legs clearly improved or disappeared, as did standing and walking alone, and ease of movement. Daily activities of living such as eating, dressing, personal hygiene and handwriting were all made easier. Clear improvements in many symptom areas were also documented by the film evidence" (Carruthers, 2004). An excerpt of the film can be seen on www.medan.pl.

The mental condition of the patients also improved substantially. The patients who had been slow, even dull, depressive, unwilling to contact with others, once the therapy commenced, changed dramatically.

After the hCG therapy was introduced, PD and other accompanying symptoms improved noticeably. Dyskinesis and involuntary body functions, being the adverse effects of treatment with L-dopa, discontinued or occurred sporadically.

If any patients had not been able to stand up, that problem disappeared. Now the patients could walk by themselves. They also reported that the way they moved had improved. Without any help of others, they could walk on an uneven ground or even stroll in woods. 
The patients were able to eat their meals without any help, they could normally use a spoon, a knife and a fork because the hand tremor stopped. They could do their shirt and coat buttons up, and needed no help from others to get dressed or to wash themselves.

Another important objective element of improvement was the disappearance of sialosis. Their handwriting also improved, became clearer, with no traces of tremor or micrographism.

In 5 out of 16 patients described here, the improvement of their quality of life (QOL) also included the sexual function. Both their libido and potency improved. They resumed sexual activity, in which they had been inactive for a long time.

The hormonal therapy enabled them to live as before they fell ill, without having to be helped by others. Their quality of life benefited from that situation too. Their caregiving families felt extremely relieved. PD patients' families voluntarily admitted that the hormonal therapy was effective not only for PD sufferers but it improved quality of life of their families as well.

Initially, the PD-treatment regime had been kept. Neither the drugs nor their doses had been changed. After a few months of hCG administration, though, it was possible to lower the dosage or even withdraw certain anti-parkinsonism drugs, without aggravation of PD symptoms.

In the study presented, there was no control group. Yet, when the hormone-stimulation therapy was stopped, the patients' condition and symptoms clearly deteriorated, returning to what they had been like before the hormonal treatment was administered. Once the hormonal therapy was restarted, parkinsonian symptoms disappeared again. That fact was observed by patients and by their families as well.

Parkinson's disease and hormonal deficiency are inextricably linked. Both men and women suffer from Parkinson's disease because of deficit of estradiol, and more specifically because of the deficit of 4-hydroxyestradiol (dihydroxyestradiol), which is a derivative of estradiol. The molecular structure drawings, which illustrate how dopamine and 4-hydroxyestradiol are attached to the same key site to dopamine receptor in the brain, as given above, are in the subsection 2.1. Late Onset Hypogonadism is not just testosterone deficiency. And it is only 4hydroxyestradiol - a chemical compound, which due to its chemical structure, may saturate dopamine E-2 receptors in the brain. Therefore, in the treatment of Parkinson's disease special attention should be paid not only to testosterone levels, but particularly to the concentration of E-2. The desirable concentration of E-2 is approximately $250-300 \mathrm{pmol} / 1$. This implies, however, maintaining also excessively high levels of testosterone, which cause hyperactivity in patients. In such cases, apart from hCG injections, I resort to microdoses of transdermal E-2 treatment in order to obtain the desired physiological serum levels of testosterone.

\subsection{LOH and mortality \& morbidity from coronary artery disease}

Cardiovascular diseases, which include CAD, are now the biggest killer in the developed countries. The mortality rate from CAD varies by a factor of five in different populations in the world. Yet, in spite of such a high range in mortality rate, the ratio of male-to female deaths is relatively constant at more than three-to-one (Tunstall-Pedoe, 1999). This cannot be explained solely by differences in the risk factors for cardiovascular disease, between the sexes (Rayner, et al., 1998). The remarkably lower prevalence of CAD is particularily prominent in pre-menopausal women, but increases slightly after the menopause (Kalin, \& Zurnoff 1990). 
These facts led to the notion that diminshing levels of testosterone in aging men, which lead to late onset hypogonadism $(\mathrm{LOH})$, are responsible for such differences and higher mortality of men. Indeed, the nested case-control study on 11'600 men aged 40 to 79 years (EPIC-Norfolk UK) showed inverse relationship of endogenous testosterone concentration and mortality due to cardiovascular disease and all causes including CAD and cancer. This study clearly suggested that high endogenous testosterone concentrations appear to lower mortality rates of men due to all causes, cardiovascular disease and cancer (Khaw, K.T., et al., 2007).

In the "Hypogonadism in Males (HIM)" study (Mulligan, et al. 2006) on 2162 male patients over 45 years of age and visiting their primary care physicians for general reasons, it was found that $38.7 \%$ of them were hypogonadal and $3.7 \%$ of them were receiving testosterone. Among men not receiving testosterone, odds ratios for having hypogonadism were significantly higher in men with arterial hypertension (1.84), hyperlipidemia (1.47), diabetes (2.09), obesity (2.38). Conversely, late onset hypogonadism (LOH) and the Testosterone Deficiency (TD) connection with cardiovascular disease is evident from TD associations with diabetes (Dhindsa, et al. 2010) and metabolic syndrome (Kalyani \& Dobs, 2000) and such findings as an inverse relationship between testosterone level and aortic atherosclerosis, which was seen among middle-aged and older men in the Rotterdam Study (Hak, et al., 2002).

Low free testosterone has also been associated with abdominal aortic aneurysm in community-dwelling men aged 70-88 years (Yeap et al., 2010). Furthermore, for men aged 50 to 91 years of age who were followed for 20 years in the Rancho Bernardo Study (BarrettConnor et al., 1999), serum testosterone levels were inversely related to weight, body mass index, arterial blood pressure, serum insulin and plasma glucose. Nevertheless, as these factors may be relevant to mortality there was clear evidence that men with serum testosterone concentration below 25th percentile had a $40 \%$ higher risk of death, independent of obesity, lifestyle choices (eg. exercise, smoking) and age (Laughlin, et al., 2008, Tivestena et al., 2009). Another 5-year retrospect study on male veterans $(\mathrm{N}=858)$, compared mortality of men with normal $( \pm 450 \mathrm{ng} / \mathrm{dL}) \mathrm{T}$ levels to those with low $(<250$ $\mathrm{ng} / \mathrm{dL})$ total testosterone and low $(<0.75 \mathrm{ng} / \mathrm{dL})$ free testosterone levels (Shores, et al., 2006). The results showed that mortality for subjects with low $\mathrm{T}$ was almost twice as high $(34.9 \%)$ as for those with normal T levels was $20.1 \%$. There is an obvious link between mortality and morbidity due to coronary CAD and other cardiovascular diseases and $\mathrm{LOH}$ due to low free testosterone levels in serum of aging men (Wu \& von Eckardstein, 2003, Wu, et al., 2010). This is due to existing strong correlation of testosterone defficiency with glucose tolerance, visceral obesity, serum lipid disorders and elevated arterial pressure (Kalyani \& Dobs, 2007). These factors usually lead to vascular disorders. The clinical studies (English, et al., (1997) showed that low natural androgen concentration can cause deletorious changes in atherogenic lipid profile, high fibrinogen and a hypercoagulable state, an increase in insulin resistance and hyperinsulinemia, higher systolic and diastolic blood pressures. The biologically plausible mechanism of testosterone protective action against cardiovascular disorers can be explained by the fact that testosterone has direct vasoactive properties (English, et al., (1997). In animal models of isolated coronary, femoral and pulmonary arteries testosterone showed a dose-dependent vasodilatory effect (Channer \& Jones, 2003). It is caused by a direct effect on the vascular smooth muscle, by either an effect on potassium or calcium channels (Deenadayalu, V.P., et al. 2001, English, K.M., 2002). In man, testosterone has been shown to cause dose-dependent vasodilation both in vitro and in vivo. During cardiac surgery on man, the coronary artery diameter and subsequent 
coronary flow immediately increased when testosterone was injected via intracoronary indwelling catheter (Webb, C.M. et al., 1999).

During the non-invasive therapy of $\mathrm{LOH}$ when the increased $\mathrm{T}$ concentration levels can be sustained for longer periods. T can not only protect man against atherogenic factors but also may cause long-term coronary dilatation. This is due to testosterone interaction with arginase activity, which results in increased synthesis of nitric oxide in endothelial cells in the entire vascular system. Nitric oxide is a well known vasodilator, which acts systemically. The synthesis of NO is also used in the treatment of erectile dysfunction (ED) by applying fosfodiesterase 5 inhibitors (PDE-5-I). Interestingly, nowadays these PDE-5 inhibitors become popular as a treatment of choice for some cardiovascular diseases.

Large scale clinical studies on long term effects of treatment of LOH with hCG on CAD were not yet reported, but case reports from my colleagues in cardiology who clinically follow some of my patients with exercise tests, are positive, including diminshing or disappearance of so called angina pectoris pain.

\subsection{Late - onset hypogonadism and immune resistance}

I have no scientific evidence to support any representation of a change in the immunity in my patients undergoing hormone therapy. Conducting such research is practically very difficult, perhaps impossible. Antibiotics, aspirin, and anti-inflammatory drugs should not be taken for at least a year by patients who have their immune status tested. This is impossible!!! But I can say with conviction that men whose testosterone levels increased 2fold during hCG therapy as well as those already experiencing androversion (for androversion see $3.3 \mathrm{LOH}$ and androversion) claimed that they did not get colds and that they had no infections, which they had had quite often before.

\section{The treatment of late - onset hypogonadism}

Now, the most widely practiced therapy for men with $\mathrm{LOH}$ are injections of testosterone esters or transcutaneous applications of $1 \%$ and $2 \%$ testosterone gel preparations. This is because many years ago it was erroneously believed that, with age, all men lose their ability for endo-synthesis of testosterone.

Another method known over the past 50 years, presently gaining "renaissance" of recognition, has been the stimulation of testosterone endo-synthesis by administering hCG (Gould, 1951, Gomula, 2001, Gomuła, 2002-a, Gomuła, 2002-b, Gomuła \& Twarkowski, 2002, Gomuła, 2006, Gomuła, 2007).

The serum testosterone concentrations observed in patients treated with hCG increased by at least 150\% and up to 200\% (Gould, 1951, Janczewski, et al., 1966, Gomuła, 2001, Gomuła, 2002-a, Liu et al., 2002, Gomuła, 2007, Gomuła \& Rabijewski, 2010).

Yet, if applying hCG makes it possible to almost double testosterone concentration, why to inject testosterone esters to men when they still have the ability for endo-synthesis of their own, natural testosterone?

The hCG therapy improves testicular function, while treatment with synthetic testosterone inhibits it. Years ago, there was a trend to produce oral contraceptives for men but this idea was soon abandoned. That oral contraceptive for men was supposed to be testosterone ester pill. The information that taking synthetic testosterone not only inhibits spermatogenesis, but also inhibits male's own testosterone endo-synthesis, is now almost labeled as "classified". In fact, after three months of taking synthetic testosterone, a man becomes 
totally infertile (WHO, 1990, Wallace, et al., 1993, Anderson \& Wu, 1996, Zhang et al., 1999, Baird, 2002, Anderson \& Waites, 2003, Si-Tian, et al., 2004). Furthermore, long-time testosterone therapy may result in irreversible testicular atrophy. From this moment on, a man becomes dependent on taking testosterone. The discontinuation of testosterone intake leads to metabolic disorders, which may cause many other diseases to develop.

\subsection{Late - onset hypogonadism and hCG therapy}

The effect of the treatment of 908 patients taking hCG was described in 2010 (Gomula \& Rabijewski, 2010). Below, I present a further study of 1200 men (age range 20-89 years; mean, 54). Mean follow-up period of the patients was over 37 months.

During the therapy with hCG $(2 \times 5000$ i.u. per week) there was an average increase in serum concentrations of total testosterone from $18.4 \mathrm{nmol} / \mathrm{L}$ to $38.59 \mathrm{nmol} / \mathrm{L}$. It was noted that during the hCG therapy, there was no increase of SHBG. To the contrary, the SHBG concentrations even showed a slight decrease. This meant that as the result of hCG treatment, free and bioavailable testosterone concentrations increased. The rise was proportional to that in total testosterone, as was reported (Fiers \& Kaufman, 1999).

There was an average increase in free testosterone concentrations from $0.0829 \mathrm{ng} / \mathrm{mL}$ $(1.98 \%)$ to $0.201 \mathrm{ng} / \mathrm{mL}(2.29 \%)$ The bio-available testosterone concentration also increased: - on average from $1.94 \mathrm{ng} / \mathrm{mL}(46.4 \%)$ to $4.71 \mathrm{ng} / \mathrm{mL}(53.6 \%)$. At the same time, there was a steady increase in the average concentration of estradiol, from $138.6 \mathrm{pmol} / \mathrm{L}$ to 280.9 pmol/L. In paralel, the average PSA level decreased by $40 \%$ (from $3.09 \mathrm{ng} / \mathrm{mL}$ to 1.83 $\mathrm{ng} / \mathrm{mL}$ ) after 37 months of therapy. These results are shown in Table 9, below.

\begin{tabular}{|l|c|c|c|c|c|c|}
\hline & $\mathrm{T}$ & T-f & T-ba & E-2 & SHBG & PSA-t \\
\hline Before & 18.4 & $0.0829(1.98 \%)$ & $1.94(46.4 \%)$ & 138.6 & 35.1 & 3.09 \\
\hline During & 38.59 & $0.201(2.29 \%)$ & $4.71(53.6 \%)$ & 280.9 & 34.86 & 1.83 \\
\hline
\end{tabular}

$\mathrm{T}=$ total testosterone (in nmol/L), $\mathrm{T}-\mathrm{f}=$ free testosterone (in $\mathrm{ng} / \mathrm{mL}$ ), $\mathrm{T}-\mathrm{ba}=$ bioavailable testosterone (in $\mathrm{ng} / \mathrm{mL}$ ), E-2=estradiol (in pmol/L), SHBG=sex hormone binding globulin (in nmol/L), PSA-t = total Prostate-Specific Antigen (in $\mathrm{ng} / \mathrm{mL}$ ).

Table 9. Testosterone (total, free, bioavailable), estradiol, SHBG \& PSA before and during/after 3 years of hCG therapy

During LOH treatment with testosterone there was also no significant PSA increase, which was regarded as evidence further supporting the safety of this therapy (Wang, et al., 2009). But achieving a $40 \%$ reduction in PSA during the hCG therapy, with a simultaneous increase of more than 2-fold in serum testosterone levels as reported above show for the first time that the hCG therapy is not only highly effective for all age groups, but quite safe as well.

There is clear evidence now that hCG therapy is safe and effective as a treatment of choice for men with late onset hypogonadism. During many years of continuous use of preparations, where hCG was the active ingredient, for LOH therapy, none of untoward side-effects were observed nor reported. It has also been confirmed by the files at the Division for Monitoring of Adverse Actions of the High Authority for Registration of Drugs and other Medical Products in Poland. The reports in these files have shown that in the last 14 years there has not been any case reporting a man showing the potential side effects of preparations used in the treatment, in which the hCG is the active ingredient (Reports from 
the years 1996-2009). This applies to 1200 patients treated by my and to the thousands of other patients treated by other physicians across Europe.

\subsection{LOH and the possibility to restore testosterone endosynthesis}

Stimulation of hormonal balance with hCG in LOH men, in most cases does increase endosynthesis of natural testosterone. This is true also for all age groups. In young men the increase can be even as high as $300 \%$. With age, the induction of endosynthesis with hCG therapy gets relatively weaker, but still effective enough to eradicate manifestations of the Testosterone Deficiency Syndrome (TDS). The analysis of the findings of the author's studies show that the opinions about the Testosterone Deficiency Syndrome etiology, so far reported in medical publications were erroneous. The author's studies on the material of 908 males treated with the hCG preparations for inducing testosterone endosynthesis, has proved that such induction is possible in any age group, but physiologically it diminishes with man's age. (Gomuła \& Rabijewski, 2010). Yet, effective endosynthesis is maintained until late senility, which is in the contrast to the belief that Leydig's cells completely cease to function with age. In men aged 30-39 years, the mean increase of serum testosterone concentration resulting from hCG therapy was $128 \%$, in those aged $40-49$ years $-126 \%$, in age group of $50-59$ years $-129 \%$, aged $60-69$ years $-121 \%$, aged $70-79$ years $-99 \%$, and in those aged 80 - 99 years it was $88 \%$.

A statistical analysis has revealed that the effectiveness of hCG therapy depends mainly on the $\mathrm{LH} / \mathrm{F}$ ratio, and to a lesser extent on the patient's age (where: $\mathrm{LH}=$ luteinizing hormone in the IU/L, and F = free PSA (expressed as free PSA/total PSA in \%).

The data showing the relationship of the decrease in the ability of testosterone endosynthesis with the LH/F ratio, rather than age, are provided in Table 10, below.

\begin{tabular}{|c|c|c|c|c|c|c|}
\hline & LH:F=0.1 & LH:F=0.15 & LH:F=0.20 & LH:F=0.25 & LH:F=0.3 & LH:F=0.35 \\
\hline $30-40 y$ & $175 \%$ & $160 \%$ & $145 \%$ & $130 \%$ & $115 \%$ & $100 \%$ \\
\hline $40-50 y$ & $164 \%$ & $149 \%$ & $134 \%$ & $119 \%$ & $104 \%$ & $89 \%$ \\
\hline $50-60 \mathrm{y}$ & $153 \%$ & $138 \%$ & $123 \%$ & $108 \%$ & $93 \%$ & $78 \%$ \\
\hline $60-70 \mathrm{y}$ & $142 \%$ & $127 \%$ & $112 \%$ & $97 \%$ & $82 \%$ & $67 \%$ \\
\hline $70-80 \mathrm{y}$ & $131 \%$ & $110 \%$ & $109 \%$ & $89 \%$ & $72 \%$ & $52 \%$ \\
\hline $80-89 \mathrm{y}$ & $119 \%$ & $98 \%$ & $84 \%$ & $81 \%$ & $60 \%$ & $41 \%$ \\
\hline
\end{tabular}

Table 10. Ability of testosterone endosynthesis versus the $\mathrm{LH} / \mathrm{F}$ ratio and age

Knowing the LH/F ratio helps to predict the outcome of the hCG treatment. When the $\mathrm{LH} / \mathrm{F}$ ratio is lower than $\mathbf{0 . 1 5}$, a patient is still able to rebuild the synthesis of endogenic testosterone. Once LH/F ratio increases above $\mathbf{0 . 3 0}$, a patient is much less likely to be able to achieve that. Another very important correlation. The serum LH and free PSA levels are essential for the health of prostate. In the recent studies of drugs for treatment of benign prostatic hyperplasia (BPH), there is an emphasis on lowering LH. For many years the LHRH analogues have been used to treat prostate cancer.

When the LH/F index in a patient with late onset hypogonadism exceeds 0.5 , there is an indication for particularly thorough detection of prostate cancer. 
The results of my follow-up of these 1200 patients show clearly that the decrease in the ability of testosterone endosynthesis is synonymous with increased risk of prostate cancer.

\subsection{Late - onset hypogonadism and androversion}

The effect of HRT with exo-testosterone is that after the treatment is discontinued, the patient's testosterone concentration level is lower than that which was when the treatment was initiated. In contrast to this, when hCG therapy is discontinued, the abilities for testosterone endo-synthesis are the same as they were before the treatment. Around $30 \%$ of patients developed a phenomenon, which can be called androversion - no need nor willingness to continue the hCG therapy, once an increase of $50-150 \%$ of testosterone levels was reached by natural endosynthesis.

The long term hCG treatment (6-24 months) can cause permanent increase (i.e. saturation) in testosterone endosynthesis, so the further induction by hCG administration will be not indicated. Is it possible to predict whether a man has the chance of achieving such a state of androversion? The answer is still uncertain. When a man, in his initial blood tests, has a very high concentration of LH and low testosterone levels, the prognosis for him to reach a state of androversion is negative. In such cases, the thorough prostate cancer detection tests are highly indicated, as such a patient belongs to the high-risk group. What is more, there is no increased PC risk associated with the continuing of hCG therapy for such a patient. Importantly, however, there may be an increased risk of prostate cancer, if and when the hCG therapy is discontinued. In case when there is a decrease in SHBG, this indicates that hCG therapy can be safely discontinued. The concentrations of LH, testosterone, SHGB, E-2, and PSA must be measured after one month and then again after two months. If for a period of two months, the concentrations of testosterone are high, in excess of those in the previous therapy, it is the proof of the onset of androversion. Furthermore, it is the sign of the end of indications for further hCG treatment. The aforementioned parameters should be monitored every 6 months. If, after 6 months, one notices a drop in serum testosterone, it is advisable to renew regular hCG treatment. If, however, a low concentration of testosterone, high LH levels, and elevated PSA reccur in the subsequent blood tests, this should be a signal to do further diagnostic tests, in order to be able to exclude or to undertake measures to treat prostate cancer.

\subsection{Recomendation for Late - onset hypogonadism therapy - discussion}

The problem with therapy of $\mathrm{LOH}$ in men, is the long-standing notion that andropause is like mirror image of menopause. Commonly, by the term «menopause » we understand the period when amenorrhea sets in for many years to follow, and women may even undergo HRT. A rather correct term for this period of women's life is " post-menopause ». The medically defined " menopause » lasts for only few months during which, the menstrual cycle gradually comes to a stop. This is due to the rapid decay and eventually total cessation of synthesis capacity of hormones in ovaries.

In men, such a phenomenon does not take place. A man loses his hormonal capacity, fertility and sexuality gradually over a period of many, many years. There are no such things in men, like such rapid, almost dramatic drops in concentrations of gonadal hormones.

The official recommendations of the National Health Service of Poland for treatments of men with $\mathrm{LOH}$, recommend the use of both: either Testosterone or hCG therapies with no emphasis on either one. This well-balanced approach merits recognition. 
Testosterone preparations - hormone replacement therapy (HRT) - are recommended in a series of guidelines for the $\mathrm{LOH}$ treatment established by the world renowned doctors and scientists. In their work, there is also reference to the HCG treatment: "Human chorionic gonadotropin (hCG) stimulates testosterone production of Leydig cells, albeit at a lower rate in older men, than in younger men. Since insufficient information exists about the therapeutic and adverse effects of hCG treatment in older men and its higher cost, this treatment cannot be recommended in $\mathrm{LOH}$, except when fertility is an issue » (Wang, et al., 2009).

Over the last almost 50 years there have been many studies published on the effectiveness of hCG to induce endosynthesis of testosterone. They showed increases of testosterone concentrations from 150\% to over 200\% resulting from hCG therapy (Gould, 1951, Gomuła, 2001, Gomuła, 2002-a, Liu, et al., 2002, Gomuła, 2007, Gomuła \& Rabijewski, 2010).

Own prospective study of 1200 patients aged from 20 to $89 \mathrm{y}$ and monitored for $>10$ years undeniably showed hCG effectiveness in inducing endosynthesis of T. In effect, the hCGtherapy resulted in the increase of serum testosterone from a mean of $18.4 \mathrm{nmol} / \mathrm{L}$ initially, to mean $38.59 \mathrm{nmol} / \mathrm{L}$ during therapy (Gomuła \& Rabijewski, 2010). There is clear evidence that hCG therapy is safe and effective as a treatment of choice for men with late onset hypogonadism. It has also been confirmed by the reports on file at the Division for Monitoring of Adverse Actions of the High Authority for Registration of Drugs and other Medical Products in Poland. The reports have shown that in the last 16 years there has not been any case reporting a man showing the potential side effects of preparations used in the treatment, in which the hCG is the active ingredient (Reports from the years 1996-2011).

This applies to 1200 patients treated by me and to the thousands of other patients treated by other doctors across Europe.

On the economic side, the treatment cost of LOH when using hCG is lower than the cost of the newly available T preparations. In the UE countries, it amounts to about 50 Euro/month. It is beyond any doubt that some authors of such consensus statement are aware of the negative impact of testosterone replacement therapy on fertility of men. They are the authors who published on the contraceptive action of $\mathbf{T}$ in men (Wallace, et al., 1993, Anderson \& Wu, 1996, Weinbauer, et al, 2001).

Furthermore, many peer reviewed papers, including the report from World Health Organization, confirmed that after up to three months of HRT with testosterone, from $40 \%$ to $90 \%$ male patients developed azoo-spermy or significant oligo-spermy - clearly a contraceptive situation - leading to a permanent pharmacological sterilization of men! (WHO, 1990, Wallace, et al., 1993, Anderson \& Wu, 1996, Zhang et al., 1999, Baird, 2002, Anderson \& Waites, 2003, Si-Tian, et al., 2004).

These facts have been known for over 20 years, but are still suppressed from information available to the general public. Just to the contrary, the mandatory information for physicians and patients attached to each box of the products, does not even mention the infertility/sterilization as a possible side-effect action of HRT with testosterone! Even in the newest-generation testosterone product ANDROTOP, the attached leaflet lists only the following warning: "The Androtop medication is not intended for treatment of male infertility or impotence.. » .

When comparing the two facts:

1. treatment of men with exo-applied synthetic testosterone may cause not only infertility but also anatomical and functional destruction of testicles and eventually leads to their complete atrophy including disappearance; 
2. treatment with hCG improves functioning of testicles, with corresponding increase of secretion of "good ", natural testosterone, the conclusion is simple: the treatment of $\mathrm{LOH}$ with naturally secreted testosterone is better, safer and more economical then it is a case synthetic exo-applied testosterone!

The superiority of the hCG treatment over the testosterone treatment has already been described (Gould, 1951).

This information, however, did not receive universal recognition. Yet hCG therapy has been proven to be effective and safe. Moreover, as a result of testosterone treatment, permanent sterilization of men follows. Other risks, which mainly occur with long-term use include irreversible testicular atrophy. And then only successful marketing of pharmaceutical companies remains. Patients seem condemned to a lifetime use of testosterone. Otherwise their cellular metabolism drops so low that they are vulnerable to many diseases and to early death.

I want to apologize to the guideline authors - some of whom are my friends - but I cannot accept the fact that not having conducted any studies on hCG treatment they ruled that the method was uncertain. They claimed that perhaps it was even dangerous. Is the lack of experience concerning the effects of hCG in the LOH treatment a sufficient reason to reject this alternative safe, effective and affordable therapy?

\section{Conclusions}

This has turned out to be the most troublesome section...

Not because I had objective problems, no, but because of my ethical issues.

Among the authors of $\mathrm{LOH}$ therapy guidelines, which were established at international levels, there are my long-time friends. I do not want to upset them because I strongly believe that what they did was acting in good faith, but I cannot exclude the fact that they had been insidiously stimulated by the pharmaceutical industry. Yet, the ultimate aim of a physician's actions is acting for the good of a patient, so in such context, the friendship takes a second place. Consequently, the conclusions that are listed below are very delicate ethically but unambiguously to the point.

1. There is no doubt now that testosterone deficit, which grows with age, negatively affects the man's biology in its broad meaning.

2. The own material presented in the paper allows the author to conclude that the minimal reference values for testosterone concentration levels, at which testosterone therapy should be initiated, which are accepted universally so far, are definitely too low.

3. Introducing a therapy to increase testosterone levels only when for many years testosterone deficit has inflicted irreversible anatomical changes, seems to be clearly delayed, and is harmful for the man's health.

4. Applying testosterone replacement therapy to a man with $\mathrm{LOH}$ makes sense only when it is impossible to provoke the man's own testosterone endosynthesis. If a man is still able to rebuild his own testosterone endosynthesis, the treatment with hCG is recommended, otherwise the testosterone replacement therapy will soon make him infertile. Moreover applying testosterone replacement therapy for a long time, will result in testicular atrophy.

5. Consequently, the guidelines and recommendations on $\mathrm{LOH}$ treatment should be verified and modified as soon as possible. 


\section{Acknowledgements}

I wish to express my sincere gratitude to my close friend - Dr Bruno Lunenfeld - for inspiring me to work in the field of the Aging Male for many years, as especially to conduct studies in the effectiveness of hCG therapy in inducing testosterone endosynthesis. I received a similar support from Prof. Alvaro Morales, for which I am very grateful too. During performing my studies, I felt a constant support from Dr. Malcolm Carruthers, Prof. Assam Yashin, Prof. Peter Sheplev, Prof. Nykola Boiko, Prof. Stefan Zgliczynski - thank you all, my friends. Walking the steep path of a professional career in science, where I have attempted to prove the safety and effectiveness of hCG therapy, I have encountered numerous enemies. I want to thank them too - their activities have made me fight for the scientific truth even more tenaciously.

\section{References}

Algarte-Genin, M., Cussenot, O., Costa, P. (2004) Prevention of prostate cancer by androgens: experimental paradox or clinical reality. Eur Urol, 46, 285-295.

Anderson, J. (2003) The role of antiandrogen monotherapy in the treatment of prostate cancer. BJU, 91, 455-462.

Anderson, R.A., Wu, F.C. (1996) Comparison between testosterone enanthate-induced azoospermia and oligozoospermia in a male contraceptive study. II. Pharmacokinetics and pharmacodynamics of once weekly administration of testosterone enanthate. J Clin Endocrinol Metab, 81, 896-901.

Anderson, R.A., Waites, G.M.H. (2003) Development of methods of male contraception: impact of the World Health Organization Task Force. Fertil. Steril., 80, 1-15.

Baird, D.T. (2002) Male contraception. Endocr Rev, 23, 735-762.

Balthazar, J., Ball, G.F. (1998) New insights into the regulation and function of brain estrogen synthase (aromataze). Trends Neurosci, 21, 243-249.

Bancroft, J. (1989) Androgens, sexuality and the aging male. in: F. Labrie, L. Proulx (edit.), Endocrinology (p. 913-916). Amsterdam: Elsevier.

Barrett-Connor, E., Von Mühlen, D.G., Kritz-Silverstein, D. Bioavailable testosterone and depressed mood in older men: the Rancho Bernardo Study. J Clin Endocrinol Metab, $84,,(2), 573-7$.

Beck, A. (1967) Depression. Clinical, experimental, and theoretical aspects. London, Staple Press.

Behre, H.M., Bohmeyer, J., Nieschlag, E. (1994) Prostate volume in testosterone-treated and untreated hypogonadal men in comparison to age-matched normal controls. Clin Endocrinol, 40, 341-349.

Bonczyk, M., Zdrojowy, R., Makota, D., Kołodziej, A. (2008) Testosterone and prostate cancer. Urol Pol, 61, 19-23.

Burries, A., Banks, S., Carter, C. et al. (1992) A long-term, prospective study of the physiologic and behavioral effects of hormone replacement in untreated hypogonadal men. J Androl, 13, 297-304.

Carani, C., Rochira, V., Faustini-Fustini, M. et al. (1999) Role of oestrogen in male sexual behaviour: insights from the natural model of aromatase deficiency. Clin Endocrinol (Oxf), 51, 517-524.

Carruthers, M. (2004) Androgen deficiency in the adult male. Taylor \& Francis, London, Oxforshire and New York. 
Channer, K.S., Jones, T.H. (2003) Cardiovascular effects of testosterone: implications of the „male menopause" Heart, 89, 121-122.

Czyżowska, A. (2009) The importance of hormone replacement therapy in sexual health and in depression for men. PhD thesis. Department of Psychology, University of Warsaw.

Davidson, J., Kwan, M., Greenleaf, W. (1982) Hormonal replacement and sexuality. Clin Endocrinol Metab, 11, 599-614.

Debruyne, F., Gres, A.,A., Arustamov, D.,L. (2008) Placebo-Controlled Dose-Ranging Phase 2 Study of Subcutaneously Administered LHRH Antagonist Cetrorelix in Patients with Symptomatic Benign Prostatic Hyperplasia. Eur Urol, 54, 170-180.

Deenadayalu, V.P., White, R.E., Stallone, J.N. et al. (2001) Testosterone relaxes coronary arteries by opening large-conductance calcium-activated potassium channel. Am J Physiol, 281, 1720-7.

Deslypere, J.,P., Vermulen, A. (1984) Leydig cell function in normal men: effects of aging, life-style, residence, diet, and activity. J Clin Endocrinol Metab, 59, 955-62.

Dhindsa, S., Miller, M.G., McWhirter, M,L, et al. 2010 Testosterone concentrations in diabetic and nondiabetic obese men. Diabetes Care, 33, 1186-1192.

Edwards, E.,Ch., Kitt, E., Oliver, J. et al. (2002) Depression and Parkinson's Disease: a new look at an old problem. Depression and Anxiety, 16, 39-43.

English, K.M, Steeds, R.P., Jones, T.H. et al. (1997) Testosterone and ischemic heart disease-is there a link? QJM, 90, 787-91.

English, K.M., Jones R.D., Jones, T.H. et al. (2002) Testosterone acts as a coronary vasodilator by a calcium-channel antagonist action. J Endocrinol Invest, 25, 455-8.

Feldman, H.A., Longcope, C., Derby, C. et al. (2002) Age trends in the level of serum testosterone and other hormones in middle-aged men: longitudinal results from Massachusetts Male Aging Study. J Clin Endocrinol Metab, 87, 589-598.

Ferrini, M., Wang, C., Hakim, A.S. et al. (2001) Age-related increased expression of inducible NDS and cytotoxic markers in T2T hypothalamic regions. Neuroendocrinology, $74,1-8$.

Ferrini, R.L., Barrett-Connor, E. (1998) Sex hormones and age: a cross-sectional study of testosterone and estradiol and their bioavailable fractions in community dwelling men. American Journal of Epidemiology, 147, 750-754.

Fiers, T., Kaufman J.M. (1999) A critical evaluation of simple methods for the estimation of free testosterone in serum. J Clin Endocrinol Metab, 84, 3666-3672.

Fossa, S., Opjordsmoen, S., Haug, E. (1999) Androgen replacement and quality of life in patients treated for bilateral testicular cancer. Eur J Cancer , 35, 1220-1225.

Fowler, J.E., Whitmore, Jr W.F. (1981) The response of metastatic adenocarcinoma of the prostate to exogenous testosterone. J Urol, 126, 372-375.

Gomula, A. (2001) The influence of chorionic gonadotropin on the endogenic testosterone synthesis. 1st Asian ISSAM Meting on the Aging Male. Kuala Lumpur. 1-4.03. 2001. Aging Male, 3: 85.

Gomula, A. (2002-a) Clinical effects of hCG treatment - new method of hormonal repleacement therapy. 3th World Congress on the Aging Male, Berlin 2002. Aging Male, 2002, 4, 260.

Gomuła, A. (2002-b) The deficit of androgens and aging men. Urol Pol, 55, 13-22. 
Gomuła, A., Twarkowski P. (2002) Effect of hormone replacement therapy for benign prostatic hyperplasia. XXXII Congress of Polish Urological Association, Urol Pol, $55,41$.

Gomula, A. (2006) The influence of sex hormones and neurotransmitters on sexual function and behaviour. Polish Sexology, 4, 21-33.

Gomuła, A. (2006) The influence of sex hormones and neurotransmitters on sexual function and behaviour. Polish Sexology, 4, 21-33.

Gomuła, A. (2007) When a man ages. Medan, Warszawa, ISBN 83-911373-2-5

Gomuła, A., Rabijewski, M. (2010) Testosterone deficiency syndrome - diagnosis and treatment - based on age-related testosterone referent levels. Polish Sexology, 8, 1-16.

Gooren, L.J.G. (2000) Quality-of-life issues in the aging male. Aging Male, 3, 185-189.

Gooren, L.J.G., Bunck, M. C. M. (2004) Androgen replacement therapy. Drugs, 64, 1861-1891.

Gould, W. (1951) The male climacteric. Medical Time, 79, 154-161.

Gould, D.C., Kirby, R.S. (2006) Testosterone replacement therapy for late onset hypogonadism: what is the risk of inducing prostate cancer? Prostate Cancer and Prostatic Diseases, 9, 14-18.

Gould, D.C., Feneley M.R., Kirby R.B. (2006) Prostate-specyfic antigen testing in hypogonadism: implications for the safety of testosterone replacement therapy. BJU International, 98, 1-9.

Hajjar, R., Kaiser, F., Morley, J. (1997) Outcomes of long-term testosterone replacement therapy in older hypogonadal males: a retrospective study. J Clin Endocrinol Metab, 82, 3793-3796.

Hak, A.E., Witteman, J.C., de Jong, F.H. et al. (2002) Low levels of endogenous androgens increase the risk of atherosclerosis in elderly men: the Rotterdam study. J Clin Endocrinol Metab, 87, 3632-3639.

Harbitz, T.B., Haugen, O.A. (1974) Endocrine disturbances in men with benign hyperplasia and carcinoma of the prostate. A morphological study in an autopsy series. Acta Path Microbiol Scand, 244, (suppl.), 1-13.).

Harman, S.M., Metter, E.J., Tobin, J.D. et al. (2001) Longitudinal effects of aging on serum total and free testosterone levels in healthy men: Baltimore Longitudinal Study of Aging. J Clin Endocrinol Metab, 86, 724-731.

Hijazi, R.A., Cunningham, G.R. (2005) Andropause: is androgen replacement therapy indicated for the aging male? Annual Review of Medicine, 56, 117-137.

Hoffman, M.A., DeWolf, W.C., Morgentaler A. (2000) Is low serum free testosterone a marker for high grade prostate cancer? J Urol, 163, 824-827.

Hogervorst, E. (2004) Testosterone and Alzheimer's disease. Aging Male, 7, 31. Abstracts of the $4^{\text {th }}$ World Congress of the Aging Male.

Hsu, J.H., Shen, W.W. (1995) Male sexual side effects associated with antidepressants: a descriptive clinical study of 32 patients. International Journal of Psychiatry in Medicine, 25, 191-201.

Huggins, C., Hodges, C.V. (1941) Studies on prostatic cancer, I: the effect of castration, of estrogen and of androgen injection on serum phosphatases in metastatic carcinoma of the prostate. Cancer Res, 1, 293-297.

Janczewski, Z., Bablom, L., Czaplicki, M. (1966) Treatment of andropause. Polish J Endocrinol $17,443-447$. 
Kalin, M.F., Zurnoff, B. (1990) Sex hormone and coronary disease: a review of the clinical studies. Steroids, 55, 330-52.

Kalyani, R.R., Dobs, A.S. (2007) Androgen deficiency, diabetes and metabolic syndrome. Curr Opin Endocrino. Obes, 2007; 14: 226-234.

Kaufman, J., T'Sjoen, G. (2002) The effects of testosterone deficiency on male sexual function. Aging Male, 5, 242-247.

Kaufmann, J.M., Vermeulen, A. (2005) The decline of androgens levels in elderly men and its clinical and therapeutic implications. Endocr Review, 26, 833-876.

Keller, A., Hamer, R., Rosen, R.C. (1997) Serotonin reuptake inhibitor-induced sexual dysfunction and its treatment: a large-scale retrospective study of 596 psychiatric outpatients. Journal of Sex and Marital Therapy, 3, 165-175.

Khaw, K.T., Dowsett, M., Folkerd, Circulation 2007.

Khosla, S., Melton, L.J., Atkinson, E.J. et al. (1998) Relationship of serum sex steroid levels and bone turnover markers with bone mineral density in men and women: a key role for bioavailable estrogen. J Clin Endocrinol Metab, 83, 2266-2274.

Kwan, M., Greenleaf, W., Mann, J. (1983) The nature of androgenom men sexuality: A combinated laboratory/self reporter study oh hypogonadal men. J Clin Endocrinol Metab, 57, 557-561.

Laughlin, G.A., Barrett-Connor, E., Bergstrom, J. Low serum testosterone and mortality in older men. J Clin Endocrinol Metab, 93, 68-75.

Lazarou, S., Reyes-Vallejo, L., Morgentaler, A. (2006) Wide variability in laboratory reference values for serum testosterone. Journal of Sexual Medicine, 3, 1085-1089.

Leder, B.Z., LeBlanc, K.M., Schoenfeld, D.A. et al. (2003) Differential effects of androgens and estrogens on bone turnover in normal men. J Clin Endocrinol Metab, 88, 204210.

Liu, P.Y. Wishart, S.M., Handelsman, D.J. (2002) A double-blind, placeb-controlled, radndomized clinical trial of recobinant human chorionic gonadotropin on muscle strenght and physical function and activity in older men with partial ege-related androgen deficiency. J Clin Endocrinol Metab, 87 (7), 3125-35.

Lunenfeld, B., Gooren, L. (2002) Textbook of men's health. New York: The Parthenon Publishing Group. ISBN 18-421401-1-6

Lunenfeld, B., Saad, F., Hoesl, C.E., (2005) ISA, ISSAM and EAU recommendations for the investigation, treatment and monitoring of late-onset hypogonadism in males: scientific background and rationale. Aging Male, 8, 59-74.

Matsumoto, A.M. (1990) Effects of chronic testosterone administration in normal men: safety and efficacy of high dosage testosterone and parallel dose-dependent suppression of luteinizing hormone, follicle-stimulating hormone, and sperm production. J Clin Endocrinol Metab, 70, 282-287.

Montorsi, F., Salonia, A., Deho, F., Cestari, A., Guazzoni, G., Di Silverio, F. (2003) Pharmacological management of erectile disfunction. B J Urol Int, 91, 446-454.

Montorsi, F. (2007) Testosterone and the Prostate: The Evidence So Far. Eur Urol, suppl., 6, 874-878.

Morales, A., Buvat, J., Gooren, L. et al. (2004) Endocrine aspects of sexual dysfunction in men. J Sex Med, 1, 69-83. 
Morales, A., Schulman, C.C., Tostain, J. et al (2006) Testosterone deficiency syndrome (TDS) needs to be named appropriately - the importance of accurate terminology. Eur Urol, 50, 407-449.

Morgentaler, A. (2006) Testosterone and prostate cancer: an historical perspective on a modern myth. Eur Urol, 50, 935-939.

Morley, J.E., Perry, III H.M. (2003) Andropause - an old concept in new clothing. Clin Geriatr Med, 19, 507-528.

Mosby's Medical, Nursing and Allied Health Dictionary. $5^{\text {th }}$ Ed., Mosby (1998), USA.

Muller, M., Grobbee, D.E., den Tonkelaar, I. et al. (2005) Endogenous sex hormones and metabolic syndrome in aging men. J Clin Endocrinol Metab, 90, 2618-2623.

Mulligan, T. Et al. (2006) Prevalence of hypogonadism in males aged at least 45 years: the HIM Study. Int J Clin Pract, 60, 762-769.

Nelson, K. (2002) Research for environmentol triggers of Parkinson's disease. The Lancet Neurol, 1, 333-41.

Nieschlag, E., Swerdloff, R., Behre, H.M. et al. (2005) Investigation, treatment and monitoring of hypogonadism in males: ISA, ISSAM and EAU recommendations. Int J Androlog, 28, 125-127.

Oesterling, J.E. (1991) LHRH Agonist. A nonsurgical treatment for Benign Prostatic Hyperplasia. J Andrology, 12, 381-388.

Official Law Daily (1996) number 121, September 11, par. 571 by Polish Ministry of Health and Social Welfare.

Okun, M.S., Walter, B.L., McDonald, W.M. (2002) Beneficial effects of testosterone replacement for the nonmotor symptoms of Parkinson disease. Arch Neurol, 11, 1750-3.

Orwoll, E., Lambert, L.C., Marshall, L.M. et al. (2006) Testosterone and estradiol among older men. J Clin Endocrinol Metab, 91, 1336-1344.

Prout, G. R., Brewer, W. R. (1967) Response of men with advanced prostatic carcinoma to exogenous administration of testosterone. Cancer, 20, 1871-1878.

Rayner, M, Mockford, C. Boaz, A. (1998) Coronary heart disease statistics. London, British Heart Foundation.

Reissmann, T., Schally, A.V., Bouchard, H et al. (2000) The LHRH Antagonist Cetrorelix: a review. Human Reproduction Update, 6, 322-331.

Reports from the years 1996-2011 the Division for Monitoring of Adverse Actions of the High Authority for Registration of Drugs and other Medical Products in Poland held by the author.

Rhoden, E.L., Morgentaler, A. (2003) Testosterone replacement therapy in hypogonadal men at high risk for prostate cancer: results of 1 year of treatment in men with prostatic intraepithelial neoplasia. J Urol, 170, 2348-2351.

Riggs, B.L. et al. (2002) Sex steroids and the construction and conservation of the adult skeleton. Endocrinological Review., 23, 279-302.

Sawada, H., Ibi, M., Kihara, T. et al. (1998) Estradiol protects mesencephalic dopaminergic neurons from oxidative stress-induced neuronal death. J Neurosci Res, 54 (5), 70719.

Schatzl, G., Madersbacher, S., Thurridl, T. et al. (2001) High-grade prostate cancer is associated with low serum testosterone levels. Prostate, 47, 52-58. 
Schiavi, R.C. et al. (1993) Hormones and nocturnal penile tumescence in healthy aging men. Archives of Sexual Behavior, 22, 207-215.

Serwin, B.B. (1994) Estrogenic effects on memory in women. Ann NY Acad, Sci, 743, 213-31.

Shabsigh, R. (2003) Hypogonadism and erectile dysfunction: the role for testosterone therapy. Int J Impot Res, 1 (supl. 4), 9-13.

Shores, M.M,, Matsumoto, A.M., Sloan, K.L.. et al., 2006 Low testosterone and mortality in male veterans. Arch Intern Med, 166, 1660-1665.

Si-Tian, L., You-Lun, G., Cui-Hong, L., Chang-Hai, H. (2004) Hormonal contraception in Chinese men: variations in suppression of spermatogenesis with injectable testosterone undecanoate and levonorgestrel implants. Asian J Androl, 6, 41-46.

Skibińska, A., Kossuth, M. (2003) Estrogens and plasticity of synapses. Neurol Neurochir Pol, Suppl. 4, 379-92.

Snyder, P., Peachey, H., Berlin, J. et al. (2000) Effects of testosterone replacement in hypogonadal men. J Clin Endocrinol Metab, 85, 2670-2677.

Snyder, P.J. (2001) Effects of age on testicular function and consequences of testosterone treatment. J Clin Endocrinol Metab, 86, 2369-2372.

Studd, J., Panay, N. (2004) Hormones and depression in women. Climacteric, 7, 338-346.

Tariq, S. (2002) Changes in libido/sex life. in: Textbook of men's health. Lunenfeld B., Gooren L. (edit.). The Parthenon Publishing Group, London, 381-388. ISBN 18-421401-1-6.

Tenover, J.L. (1997) Testosterone and the aging male. Journal of Andrology, 18, 103-106.

Tenover, J.L. (1999) Testosterone replacement therapy in older adult men. International Journal of Andrology, 22, 300-306.

Tivesten, A., Vandenput, L., Labrie, F., et al. Low serum testosterone and estradiol predict mortality in elderly men. J Clin Endocrinol Metab, 2009, 94,,2482-2488.

Traish, A.M., Kim, N. (2005) The physiological role of androgens in penile erection: regulating of corpus cavernosum structure and function. Journal of Sexual Medicine, 2, 759-770.

Trinick, T.R, Feneley M.R., Carruthers M. (2011) International web survey shows high prevalence of symptomatic testosterone deficiency in men. The Aging Male, 14, 1015.

Tunstall-Pedoe, H., Kuulasmaa, K., Mahonen, M., et al. (1999) Contribution of trends in survival and coronary event rate to change in coronary heart disease mortality. 10year results WHO MONICA Lancet, 353, 1547-57.

Vermeulen, A. (2001) Androgen replacement therapy in the aging male - a critical evaluation. J Clin Endocrinol Metab, 86, 2380-2389.

Vermeulen, A., Kaufman, J.M., Giagulli, V.A. (1996) Influence of some biological indexes on sex hormone binding globulin and androgen levels in aging or obese males. J Clin Endocrinol Metab, 81, 1821-1826.

Wallace, E.M., Gow, S.M., Wu, F.C. (1993) Comparison between testosterone enanthateinduced azoospermia and oligozoospermia in a male contraceptive study. I. Plasma luteinizing hormone, follicle stimulating hormone, testosterone, estradiol, and inhibin concentrations. J Clin Endocrinol Metab, 77, 290-293.

Wang, C., Alexander, G., Berman, N. et al. (1996) Testosterone replacement therapy improves mood in hypogonadal men - a clinical research center study. J Clin Endocrinol Metab, 81, 3578-3583. 
Wang, C., Nieschlag, E., Swerdloff, R. et al. (2009) Investigation, Treatment and Monitoring of Hypogonadism in Males: ISA, ISSAM, EAU, EAA and ASA Recemendation. Eur Urol, 55, 121-130.

Webb, C.M., MacNeil, J.G., Hayward C.S. et al. (1999) Effects of testosterone on coronary vasomotor regulation in men with coronary heart disease. Circulation, 100, 1690-6.

Weinbauer, G.F., Schlatt, S., Walter, V., Nieschlag, N. (2001) Testosterone-induced inhibition of spermatogenesis is more closely related to suppression of FSH than to testicular androgen levels in the cynomolgus monkey model (Macaca fascicularis). J Endocrinol, 168, 25-38.

World Health Organization Task Force on Methods for the Regulation of Male Fertility. Contraceptive efficacy of testosterone-induced azoospermia in normal men. Lancet (1990), 336, 955-959.

Wu, F.C., von Eckardstein, A. (2003) Androgens and coronary artery disease. Endocr Rev, 24, 183-217.

Wu, F.C., Tajar, A., Beynon, J.M., et al. (2010) EMAS Group. Identification of late-onset hypogonadism in middle-aged and elderly men. N Engl J Med, 363, 123-135.

Yano, M, Imamoto, T, Suzuki, H et al. (2007) The clinical potential of pretreatment serum testosterone level to improve the efficiency of prostate cancer screening. Eur Urol, 51, 375-380.

Yassin A., Treish A. (2004) LUTS/BPH, alpha-adrenoreceptors and erectile function. Arab Journal of Urology, 2, 8-12.

Yeap, B B , Hyde, Z., Norman, P.E., Chubb, S.A.P., Golledge, J. (2010) Associations of total testosterone, sex hormone-binding globulin, calculated free testosterone, and luteinizing hormone with prevalence of abdominal aortic aneurysm in older men. $J$ Clin Endocrinol Metab, 95, 1123-1130.

Zgliczyński, S. \& Zgliczyński, W. (2002) Standards of Endocrinology. Studio PIN, Warszawa, ISBN: 83-915669-1-9.

Zhang, G.Y., Gu, Y.Q., Wang, X.H., Cui, Y.G., Bremner ,W.J. (1999) A clinical trial of injectable testosterone undecanoate as a potential male contraceptive in normal Chinese men. J Clin Endocrinol Metab, 84, 3642-3647.

Zitzmann, M., Nieschlag, E. (2006) Testosterone substitution: current modalities and perspectives. J Reproduktionsmed Endokrinol, 3 (2), 109-116.

Zverina, J., Hampl, R., Sulocava, J., Starka ,L. (1990) Hormonal status and sexual behaviour of 16 men after surgical castration. Arch Ital Urol Nefrol Androl, 62, 55-58. 


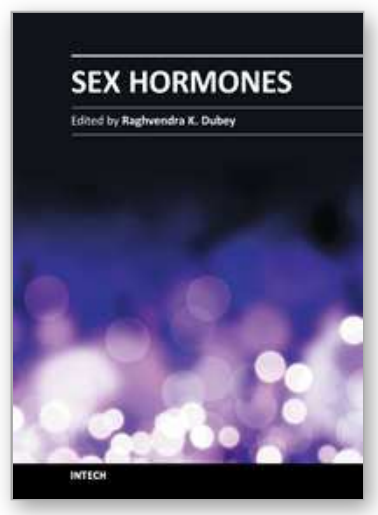

\section{Sex Hormones}

Edited by Prof. Raghvendra Dubey

ISBN 978-953-307-856-4

Hard cover, 430 pages

Publisher InTech

Published online 08, February, 2012

Published in print edition February, 2012

Sex Hormones not only regulate reproductive function, but they also play a prominent role in the biology and physiology of several organs/tissues and in the pathophysiology of several diseases. During the last two decades, the information on the mechanisms of action of sex hormones, such as estrogens and androgens, has rapidly evolved from the conventional nuclear receptor dependent mechanisms to include additional nonnuclear, non-genomic and receptor-independent mechanisms. This highlights the need to update the current knowledge on sex hormones and their mode of action. Increasing evidence that exogenous/epigenetic factors can influence sex hormone production and action highlights the need to update our knowledge on the mechanisms involved. This book provides a systematic and updated overview of the male/female sexhormones and their impact in the biology and physiology of various organs. Additionally, the book discusses their positive and negative association with the pathophysiology of various diseases (e.g. osteoporosis, cardiovascular-disease, hypogonadism, reproduction, cancer) and their therapeutic potential.

\section{How to reference}

In order to correctly reference this scholarly work, feel free to copy and paste the following:

Andrzej Gomuła (2012). Late - Onset Hypogonadism - New Point of View, Sex Hormones, Prof. Raghvendra Dubey (Ed.), ISBN: 978-953-307-856-4, InTech, Available from: http://www.intechopen.com/books/sexhormones/late-onset-hypogonadism-new-point-of-view

\section{INTECH}

open science | open minds

\section{InTech Europe}

University Campus STeP Ri

Slavka Krautzeka 83/A

51000 Rijeka, Croatia

Phone: +385 (51) 770447

Fax: +385 (51) 686166

www.intechopen.com

\section{InTech China}

Unit 405, Office Block, Hotel Equatorial Shanghai No.65, Yan An Road (West), Shanghai, 200040, China 中国上海市延安西路65号上海国际贵都大饭店办公楼405单元 Phone: +86-21-62489820

Fax: $+86-21-62489821$ 
(C) 2012 The Author(s). Licensee IntechOpen. This is an open access article distributed under the terms of the Creative Commons Attribution 3.0 License, which permits unrestricted use, distribution, and reproduction in any medium, provided the original work is properly cited. 